\title{
Photometric Light Curves and Polarization of Close-in Extrasolar Giant Planets
}

\author{
S. Seager円, B. A. Whitney?, D. D. Sasselor|3t
}

\begin{abstract}
The close-in extrasolar giant planets [CEGPs], $\lesssim 0.05$ AU from their parent stars, may have a large component of optically reflected light. We present theoretical optical photometric light curves and polarization curves for the CEGP systems, from reflected planetary light. Different particle sizes of three condensates are considered. In the most reflective case, the variability is $\approx 100$ micromagnitudes, which will be easily detectable by the upcoming satellite missions MOST, COROT, and MONS, and possibly from the ground in the near future. The least reflective case is caused by small, highly absorbing grains such as solid $\mathrm{Fe}$, with variation of much less than one micromagnitude. Polarization for all cases is lower than current detectability limits. We also discuss the temperature-pressure profiles and resulting emergent spectra of the CEGP atmospheres. We discuss the observational results of $\tau$ Boo b by Cameron et al. (1999) and Charbonneau et al. (1999) in context of our model results. The predictions - the shape and magnitude of the light curves and polarization curves - are highly dependent on the size and type of condensates present in the planetary atmosphere.
\end{abstract}

Subject headings: planetary systems — radiative transfer — stars: atmospheres

\section{Introduction}

The discovery of the planet $51 \mathrm{Peg} \mathrm{b}$ in 1995 (Mayor \& Queloz 1995), only $0.051 \mathrm{AU}$ from its parent star, heralded an unexpected new class of planets. Due to gravitational selection effects, several more Jupiter-mass close-in extrasolar giant planets (CEGPs) have been discovered since that time (Butler et al. 1997, 1998; Mayor et al. 1999; Mazeh et al. 2000). To date there are 5 extrasolar giant planets $\lesssim 0.05$ AU from their parent stars, and an additional $9 \lesssim 0.23 \mathrm{AU}$ (see Schneider 2000). Relevant data about the close-in planet-star systems (orbital distance $\lesssim 0.05 \mathrm{AU}$ ) are listed in Table 1. Ongoing radial velocity searches will certainly uncover more CEGPs in the near future. The CEGPs are being bombarded by radiation from their parent stars, and could be very bright in the optical. At best the CEGPs could be four to five orders of magnitude fainter than their primary star, compared to Jupiter which is 10 orders of magnitude fainter than the Sun.

The recent transit detection of HD 209458 b by Charbonneau et al. (2000) and Henry et al. (2000b) confirms that the CEGPs are gas giants, gives the planet radius, and fixes the orbital inclination, which removes the $\sin i$ ambiguity in mass and provides the average planet density. HD 209458 has

\footnotetext{
${ }^{1}$ Institute for Advanced Study, Olden Lane, Princeton, NJ 08540

${ }^{2}$ Space Science Institute, 3100 Marine St., Suite A353, Boulder, CO 80303-1058

${ }^{3}$ Astronomy Department, Harvard University, 60 Garden Street, Cambridge, MA 02138

${ }^{4}$ Alfred P. Sloan Research Fellow
} 
$R_{*}=1.2 \pm 0.1 R_{\odot}$ and $R_{P}=1.40 \pm 0.17 R_{J}$ (Mazeh et al. 2000), where $R_{*}$ is the stellar radius and $R_{P}$ is the planet radius. Transits are definitely ruled out for $\tau$ Boo b, 51 Peg b, $v$ And b, HD 187123, and $\rho^{1}$ Cnc $\mathrm{b}$, whether they are assumed to be gas giants with radius $1.2 R_{J}$, or smaller rocky planets with radius $\sim 0.4$ $R_{J}$ (Henry et al. 2000a, 1997; Baliunas et al. 1997; G. Henry, private communication). Transits are also ruled out for HD 75289 (M. Mayor, private communication). For a transit to be observable, a CEGP must be aligned with the star as seen from Earth with an inclination $i>\theta_{T}$, where $\theta_{T}=\cos ^{-1}\left(\left(R_{*}+R_{P}\right) / D\right)$. For random orientations, the probability for $i$ to be between $90^{\circ}$ and $j^{\circ}$ is $P(j)=\cos (j)$. With $\theta_{T} \sim 83^{\circ}$, the CEGPs have transit probabilities of ten percent. By the same $\theta_{T}$ criterion, the non-detection of transits puts limits on the orbital inclinations to approximately $83^{\circ}$ (for $R_{*}=1.16 R_{\odot}, R_{P}=1.2 R_{J}$, and $D=0.051 \mathrm{AU}$ ). Several groups (e.g. STARE (PI T. Brown), Vulcan Camera Project (PI W. Borucki), WASP (PI S. Howell)) are monitoring thousands of stars without known planets, searching with high precision photometry for periodic fluctuations indicative of a planetary transit. Follow-up observations by radial velocity techniques (or astrometry in the future) will be needed to fix the orbital radius in order to determine the planet mass. Edge-on CEGP systems are the most promising for reflected light signals.

Several observational approaches to detecting and characterizing CEGP atmospheres have been developed. These include spectral separation, transmission spectra observations during transit, infrared observations, and optical photometric light curve observations.

Charbonneau et al. (1999) and Cameron et al. (1999) have developed a direct detection technique: a spectral separation technique to search for the reflected spectrum in the combined star-planet light. Both groups have observed the $\tau$ Boo system. $\tau$ Boo A is one of the brightest (fourth magnitude), hottest (F7V) parent stars, and $\tau$ Boo b has one of the smallest semi-major axes; these three properties make $\tau$ Boo a promising candidate for this technique. From a non-detection, Charbonneau et al. (1999) have put upper limits on the planet-star flux ratio ranging from $5 \times 10^{-5}$ for $\sin i \sim 1$ to $1 \times 10^{-4}$ for $\sin i \sim 0.5$. The result is within the strict assumptions that the light curve is fairly isotropic and that the reflected spectrum is an exact copy of the stellar spectrum from 4668 to $4987 \AA$. Their upper limit on the geometric albedo is 0.3 for $\sin i \sim 1$. The same technique for the $\tau$ Boo system has been used by Cameron et al. (1999) who claim a possible detection at an inclination of $29^{\circ}$, and give a planet-star flux ratio of $1.9 \times 10^{-4}$ at $i=90^{\circ}$. Given $R_{P}$, the albedo derived from this type of observation can provide a weak constraint on theoretical models.

A second approach is to observe transmission spectra during a planet transit. The stellar flux will pass through the optically thin part of the planet atmosphere. Theoretical predictions show the planetary absorption features will be at the $10^{-4}$ to $10^{-3}$ level (Seager \& Sasselov 2000). Successful observations will constrain the cloud depth and may give important spectral diagnostics such as the presence of $\mathrm{CH}_{4}$ which is a good temperature indicator for the upper atmosphere layers.

A third technique under development is the use of the Keck infrared interferometer in the differential phase mode to directly detect and spectroscopically characterize the CEGPs. The technique is based on the difference between the very smooth infrared stellar spectrum and the strong water absorption bands and possibly methane bands in the CEGP's infrared spectrum. See Akeson \& Swain (1999) for more details.

In this paper we present theoretical photometric light curves and polarization curves of the CEGP systems. As the planet orbits the star, the planet changes phase as seen from Earth. The planet and star are too close together for their light to be separated, but this small separation means the stellar flux hitting the planet is large, and the reflected light variation in the combined light of the system from the planet's different phases may be detectable. We focus on the optical where there is a clear signature of reflected light: the planet's dark side has no reflection or emission in optical light. In contrast, there is 
no large light variation in the infrared where the CEGPs are bright on both the day and night side from reemission of absorbed light. Scattered light is minimal in the infrared, and is difficult to disentangle from the emitted light. Unlike transits, which can only be seen for inclinations $>\theta_{T}$, the reflected light curves of lower inclinations are theoretically visible and may be detectable. This work is motivated by upcoming microsatellites MOST ( 2002) (Matthews 1997), COROT ( 2003) (Baglin 1998, 2000), and MONS ( 2003) (Christensen-Dalsgaard 2000). Initially intended for asteroseismology, these satellites have capabilities to detect $\mu$ mag variability. MOST will observe known stars in a broad visual waveband, one at a time for a period of roughly one month, including one with a known CEGP in its first year. COROT's exoplanet approach will use two CCDs in two colors to observe several fields of $\sim 6000$ stars for a few months each. Because of this wide-field approach the stars with known CEGPs will not be observed by COROT. While COROT's main exoplanet focus is on transits, probability estimates suggest several CEGP light curves from reflection should be detected. Precision of ground-based photometry on the CEGP parent stars is currently at $100 \mu \mathrm{mag}$, and could reach $50 \mu \mathrm{mag}$ in the near future with dedicated automatic photometric telescopes (Henry et al. 2000a). We also present polarization signatures although they are well under the current limits of detectability which is a few $\times 10^{-4}$ in fractional polarization of the system (e.g. Huovelin et al. 1989).

This paper, to our knowledge, is the first to describe photometric light curves and polarization of CEGP systems: gas giants in close orbits around Sun-like stars. Although our own Solar System planets have been well studied in reflected and polarized light, the CEGPs have effective temperatures an order of magnitude higher, so completely different cloud species and atmospheric parameters are expected. If observable, the light curves would roughly constrain the type and size distribution of condensates in the planetary atmosphere. In $\S 2$ we present definitions and analytical estimates of reflected light from the CEGPs, in $\S 3$ a description of our model, and in $\S 4$ results and discussion.

\section{Analytical Estimate of the Light Curves and Polarization}

An analytical estimate of the amount of reflected light and polarization of an EGP system is useful for both comparison with simulations and for upper limit predictions. A good idealized case for such estimates is provided by modeling a planet as, for example, a Lambert sphere. The Lambert sphere derives from the law of diffuse reflection proposed by Lambert, postulating a reflecting surface with a reflection coefficient that is constant for all angles of incidence. The reflection coefficient is simply the ratio of the amount of light diffusely reflected in all directions by an element of the surface to the incident amount of light which falls on this element. The general conditions postulated by Lambert, for example angle independence, are satisfied strictly only for an absolute blackbody and an ideal reflecting surface (often called "absolutely white", "ideally matted", etc.). Thus derives Lambert's definition of albedo, with its inherent ambiguities as discussed at the end of the 19th century by Seeliger, and the ultimate decision by Russell (1916) to endorse Bond's (1861) definition of albedo for use in the Solar System.

The arguments offered by Russell (1916) in favor of the Bond albedo (over other albedo definitions in use at the time) are still relevant for Solar System objects, but not necessarily for EGPs. One important point in favor of the Bond albedo was that it is derivable from observations. The Bond albedo is defined as the ratio of the total amount of reflected light to the total amount of incident plane-parallel light integrated over all angles. Note that at the time of Russell - before multi-wavelength observations of the Solar System planets - the Bond albedo, $A$, was not defined as an integrated quantity over all wavelengths as it is today. However, the discussion below is still valid with either definition. The Bond albedo $A$ can be 
separated into two quantities,

$$
A=p q,
$$

where $p$ is the geometric albedo and $q$ is the phase integral. The geometric albedo is defined as the planet flux divided by the reflected flux from a perfectly diffusing disk of the same radius. The phase integral $q$ is defined as

$$
q=\int_{0}^{\pi} \phi(\alpha) \sin \alpha d \alpha,
$$

where $\phi(\alpha)$ is the phase function, or the brightness variation of the planet at different phases. The phase angle, $\alpha$, is the angle between the star and Earth as seen from the planet; $\alpha=0$ corresponds to "opposition" when the planet is maximally illuminated as seen from Earth. $p$ is measureable for all Solar System planets because it is a geometric and photometric quantity. $q$ is measureable from Earth for Mercury, Venus, Mars, and the Moon; for the outer planets whose phase angle variation is only up to several degrees from Earth satellite mission observations were necessary. Thus the benefit of the Bond albedo: it is a physically meaningful quantity but it can be determined empirically for the Solar System planets.

In contrast, the Bond albedo cannot be determined from observations for extrasolar planets. Because the EGP systems are so distant from Earth, only the CEGPs have prospects for measurement of $p$ in the foreseeable future, and even then only the most reflective CEGPs will be bright enough, and only $~ 10 \%$ of those will have orbital inclinations near 90. Charbonneau et al. (1999) and Cameron et al. (1999) have developed a spectral separation detection technique which can put upper limits on - and in the best case measure - $p$ in a narrow wavelength region. For CEGPs that are reflective, and at $i \sim 90^{\circ}, q$ should be measureable with the upcoming satellite missions. However if a given CEGP system is at $i<90^{\circ}$, the full range of phases will not be visible (i.e. $\alpha$ will not be fully probed), and $p$ and $q$ will not be measureable. As discussed in $\$ 4.7 .2$, EGPs beyond $D=0.1$ AU will not be detectable in optically reflected light even with the upcoming satellite missions. The EGPs certainly have promise for detection in the infrared where they emit most of their energy. However, most of this energy is reprocessed absorbed energy; it is not possible to measure the Bond albedo with infrared observations. To summarize, the Bond albedo came into standard usage because it was a measureable quantity. This is not possible for almost all of the EGPs because of the distance of the systems and random orbital inclinations.

The goal of this paper is a presentation of light curves at all viewing angles and at different inclinations, instead of a Bond albedo. We begin with the analytical estimate, where in the idealized case we are simply interested in the ratio, $\epsilon$, of the observed flux at Earth from the EGP at full phase $(\alpha=0)$ to that of the star: $\epsilon=p\left(R_{P} / D\right)^{2}$. Here $D$ is the star-planet distance, and $p$ and $R_{P}$ are as previously defined. For a Lambert sphere the single scattering albedo $\tilde{\omega}=1$; no photons are absorbed, and so $A=1$. For a Lambert sphere, all incoming photons are singly, isotropically scattered, and $p=2 / 3$. The light variation of the Lambert sphere is only due to phase effects - the phase function, $\phi$, is simply (Russell 1916):

$$
\phi(\alpha)=\frac{\sin (\alpha)+(\pi-\alpha) \cos (\alpha)}{\pi}
$$

and the phase-dependent flux ratio is $\epsilon \phi(\alpha)$. Note that the phase angle, $\alpha$, is a function of the orbital phase and inclination. We convert this flux ratio to variation in micromagnitudes by

$$
\Delta m=-2.5 \log _{10}(1+\epsilon \phi(\alpha)) 10^{6}
$$

Figure 1 a shows the photometric light variation at $i=90^{\circ}$ for a Lambert sphere of $R=1.2 R_{J}$ at various $D$ corresponding to known CEGP systems. The large variation of 110 and $140 \mu$ mag for planets 
with $D$ corresponding to $\tau$ Boo b and HD 187123 respectively is above current ground-based limits (Henry et al. 2000a). The Lambert sphere light curve is unrealistic, and the point of this paper is to show that the situation is far more complex and almost always conducive to a smaller light variation for a few reasons. Firstly, the single scattering albedo, $\tilde{\omega}$, is generally different from one. When optical photons are absorbed by condensates or gas in the CEGP atmospheres, they are re-emitted in the infrared or contribute to the thermal pool. Secondly, multiple scattering gives more of a chance for absorption over single scattering for the same single scattering albedo. Each time a photon scatters, its next encounter has a scattering probability of $\tilde{\omega}$, but when a photon is absorbed it cannot contribute to the scattered light. This effect depends on density, i.e. on the mean free path of the photon. Thirdly, when particles are large compared to the wavelength of light, the particle scatters preferentially in the forward direction. In this case, photons that enter the atmosphere are likely to be multiply scattered down into the atmosphere and eventually absorbed, rather than to be backscattered and escape the planetary atmosphere.

Figure $1 \mathrm{~b}$ shows the fractional polarization of the total light of a Lambert sphere at $i=90^{\circ}$ at various $D$ corresponding to known CEGP systems, and assuming $R_{P}=1.2 R_{J}$. We assume Rayleigh scattering linear polarization of unpolarized incident light $P o l_{R a y}=\sin ^{2} \theta_{S} /\left(1+\cos ^{2} \theta_{S}\right)$ (Chandrasekhar 1960). Here $\theta_{S}$ is the scattering angle: $\theta_{S}=0^{\circ}$ is the forward direction and $\theta_{S}=180^{\circ}$ the backward direction. Pol $_{R a y}$ peaks at a scattering angle of $90^{\circ}$. For single scattering and $\tilde{\omega}=1$, all scattered light is polarized. The polarization signature plotted in Figure $\mathbb{1} \mathrm{b}$ does not peak at $\alpha=90^{\circ}$ because the polarization is modulated by the reflected light curve; the scattered light is maximally polarized at $\alpha=90^{\circ}$, but the amount of scattered light peaks at $\alpha=0^{\circ}$. Plotted is $\operatorname{Pol}=\left(S_{\perp}-S_{\|}\right) /(S+F)=\epsilon \phi(\alpha) P_{\text {ol }}$ Ray , where $S$ is the total scattered light, $F$ is the unpolarized stellar flux, $S \ll F$, and $S_{\perp}$ and $S_{\|}$are the perpendicular and parallel components of the scattered light respectively. In reality polarization is much lower than this best case estimate, for a few reasons. Firstly, the amount of scattered light is expected to be lower as described above. Secondly, for the case of multiple scattering not all of the scattered light is polarized - multiple scatterings mean the photon loses some of its polarization signature. Thirdly, when the particle is large compared to the wavelength of light, different light paths through the particle and interference effects cause the polarized light to be lower and to have more than one peak, and so a strong single peaked signal such as shown in Figure it may not be reached.

As shown in Figures 1 a and $1 \mathrm{~b}$, the light curves and polarization are very sensitive to $D$, since $\epsilon \sim 1 / D^{2}$. For example, the Lambert sphere at $D=0.042 \mathrm{AU}$ (corresponding to HD 187123) has a light curve and polarization curve with amplitude twice as high as a Lambert sphere at $D=0.059 \mathrm{AU}$ (corresponding to $v$ And $\mathrm{b}$ ). The light curve and polarization curve estimates are also sensitive to $R_{P}$. Both can be scaled - the light curve approximately and the polarization curve exactly - for different $R_{P}$ by the factor $\left(R_{P} / 1.2 R_{J}\right)^{2}$. For HD $209458 \mathrm{~b}$ with $R_{P}=1.40 \pm 0.17 R_{J}$ (Mazeh et al. 2000), this factor is 1.36 .

\section{Model Atmosphere}

The model atmosphere code consistently solves for the planetary emergent flux and temperaturepressure structure by simultaneously solving hydrostatic equilibrium, radiative and convective equilibrium, and chemical equilibrium in a plane-parallel atmosphere, with upper boundary condition equal to the incoming radiation. The code is described in Seager (1999) and is improved over our code described in Seager \& Sasselov (1998) in two major ways. One is a Gibbs free energy minimization code to calculate equilibrium abundances of solids and gases, the second is condensate opacities for 3 solid species. So while in Seager \& Sasselov (1998) we considered neither the depletion of $\mathrm{TiO}$ nor accurate formation of $\mathrm{MgSiO}_{3}$, 
in the new models we do.

We compute the photometric light curves and polarization curves with a 3D Monte Carlo code (Whitney, Wolff, \& Clancy 1999), using the atmospheric profiles (opacities and densities as a function of radial depth) generated by the Seager \& Sasselov code. While in principle one could compute light curves from the model atmosphere code described above, the Monte Carlo scheme can treat a much more sophisticated scattering method, with anisotropic scattering and polarization, than our model atmosphere program. Both are described in this section.

\subsection{Chemical Equilibrum}

The equation of state is calculated using a Gibbs free energy minimization method, originally developed by White, Johnson, \& Danzig (1958), and followed up by a number of papers in the 1960s and 1970s including a particularly useful one by Eriksson (1971). A detailed description of this method can be found in those papers; more recent treatments relevant for astrophysics are described in Sharp \& Huebner (1990), Petaev \& Wood (1998), and Burrows \& Sharp (1999). A more complete chemical equlibrium calculation applied to Gliese 229 B is described in Fegley and Lodders (1996).

We have selected the most important species from Burrows and Sharp (1999) for brown dwarfs and from Allard \& Hauschildt (1995) for cool stars. We used fits to the Gibbs free energy from Sharp \& Huebner (1990), from Falkesgaard (private communication), or fitted from the JANAF tables (Chase 1998) following the normalization procedure described in Sharp \& Huebner (1990). We include ions using a charge conservation in place of the usual mass balance constraint (equation (10) in Sharp \& Huebner 1990). In the Gibbs method we include 27 elements, with 90 gaseous species and 4 solid species: H, He, O, C, Ne, N, $\mathrm{Mg}, \mathrm{Si}, \mathrm{Fe}, \mathrm{S}, \mathrm{Ar}, \mathrm{Al}, \mathrm{Ca}, \mathrm{Na}, \mathrm{Ni}, \mathrm{Cr}, \mathrm{P}, \mathrm{Mn}, \mathrm{Cl}, \mathrm{K}, \mathrm{Ti}, \mathrm{Co}, \mathrm{F}, \mathrm{V}, \mathrm{Li}, \mathrm{Rb}, \mathrm{Cs}, \mathrm{CO}, \mathrm{H}_{2}, \mathrm{OH}, \mathrm{SH}, \mathrm{N}_{2}, \mathrm{O}_{2}$, $\mathrm{SiO}$, TiO, SiS, $\mathrm{H}_{2} \mathrm{O}, \mathrm{C}_{2}, \mathrm{CH}, \mathrm{CN}, \mathrm{CS}, \mathrm{SiC}, \mathrm{NH}, \mathrm{SiH}, \mathrm{NO}, \mathrm{SN}, \mathrm{SiN}, \mathrm{SO}, \mathrm{S}_{2}, \mathrm{C}_{2} \mathrm{H}, \mathrm{HCN}, \mathrm{C}_{2} \mathrm{H}_{2}, \mathrm{CH}_{4}, \mathrm{AlH}$, $\mathrm{AlOH}, \mathrm{Al}_{2} \mathrm{O}, \mathrm{CaOH}, \mathrm{MgH}, \mathrm{MgOH}, \mathrm{VO}, \mathrm{VO}_{2}, \mathrm{CO}_{2}, \mathrm{TiO}_{2}, \mathrm{Si}_{2} \mathrm{C}, \mathrm{SiO}_{2}, \mathrm{FeO}, \mathrm{FeS}, \mathrm{NH}_{2}, \mathrm{NH}_{3}, \mathrm{CH}_{2}, \mathrm{CH}_{3}$, $\mathrm{H}_{2} \mathrm{~S}, \mathrm{KOH}, \mathrm{NaOH}, \mathrm{NaCl}, \mathrm{NaF}, \mathrm{KCl}, \mathrm{KF}, \mathrm{LiCl}, \mathrm{LiF}, \mathrm{CsCl}, \mathrm{CsF}, \mathrm{H}^{+}, \mathrm{H}^{-}, \mathrm{H}_{2}^{-}, \mathrm{H}_{2}^{+}, \mathrm{Na}^{-}, \mathrm{K}^{-}, \mathrm{Li}^{-}, \mathrm{Cs}^{-}, \mathrm{Fe}$ (solid), $\mathrm{CaTiO}_{3}, \mathrm{Al}_{2} \mathrm{O}_{3}, \mathrm{MgSiO}_{3}$.

While a full treatment of all naturally occuring elements and $\sim 2500$ compounds (e.g. Fegley \& Lodders 1996) is possible, our abridged choice is certainly adequate for a first prediction of CEGP photometric light curves. There may be non-equilibrium chemistry involved (e.g. photochemistry on our own Solar System planets) that is not addressed by even a complete thermodynamical equilibrium calculation. In general, as the temperature decreases from the inner atmosphere to the outer atmosphere, the metal gases are depleted into solids which are efficient absorbers or reflectors. The three condensate opacities we chose (solid Fe, $\mathrm{MgSiO}_{3}$, and $\mathrm{Al}_{2} \mathrm{O}_{3}$ ) have very different optical constants (see $\$ 3.3$ and $\$ 4.2$ ), and are among the dominant solids expected at the relevant temperatures and pressures.

\subsection{Radiative Transfer}

The flux from the parent star travels through the planetary atmosphere, interacting with absorbers and scatterers in a frequency-dependent manner. In a condensate-free CEGP atmosphere (Seager \& Sasselov 1998), blue light will Rayleigh scatter deep in the atmosphere where the density of scatterers is highest, while infrared light will be absorbed high in the atmosphere due to strong absorbers such as $\mathrm{TiO}$ and $\mathrm{H}_{2} \mathrm{O}$. 
Similar results were found for dusty models by Marley et al. (1999), although they considered an isolated planet of the equilibrium effective temperature. In other words, they assumed that the absorbed stellar flux can be accounted for as thermalized intrinsic flux for the calculation of the atmospheric structure. For differences in the self-consistent treatment of irradiation and an isolated planet at the same $T_{\text {eff }}$, see Seager \& Sasselov (1998) and Seager (1999).

The equilibrium effective temperature is defined by $T_{\text {eq }}=T_{*}\left(R_{*} / 2 D\right)^{1 / 2}[f(1-A)]^{1 / 4}$. Here the subscript * refers to the parent star, $D$ is the star-planet distance, $A$ is the Bond albedo, $f=1$ if the heat is evenly distributed, and $f=2$ if only the heated side reradiates the energy. Physically, $T_{\text {eq }}$ is the effective temperature attained by an isothermal planet (after it has reached complete equilibrium with its star).

Our approach is to use $F_{*}=\sigma T_{*}^{4} R_{*}^{2} / 4 D^{2}$ as the incoming flux and assume that $f=1$, as it will for planets with a thick atmosphere, due to rapid zonal and meridional circulation patterns (Guillot et al. 1996). The factor of 4 is due to the assumption that the absorbed incoming radiation is efficiently distributed to all parts of the planet: radiation incoming to a cross section of $\pi R_{P}^{2}$ is reemitted into $4 \pi R_{P}^{2}$. With this approach we need not use $T_{\text {eq }}$, nor the Bond albedo used in the $T_{\text {eq }}$ definition. Heating of the planet happens in a frequency- and depth-dependent manner, and the heating, as well as the planet's $T_{\text {eff }}$, comes out of the model atmosphere solution. We treat the incoming flux as plane-parallel, which is accurate for isotropic scattering (see $\$ 4.3 .2$ ).

An additional but small contribution to the $T_{\text {eff }}$ is the internal planetary heat. Because of the strong irradiation, the internal temperature of the planet is greater than the internal temperature of an isolated planet (Guillot et al. 1996). The planet possesses an intrinsic luminosity because it leaks some of the heat acquired during formation by loss of gravitational energy. Thus, the atmosphere's inner boundary condition is age- and mass-dependent, and needs a self-consistent atmospheric and evolutionary calculation with accurate irradiation and spectral modeling. In any case the reflected spectra are not affected by the lower boundary condition; any good guess is too cool to produce light in the optical, which is entirely reflected light.

We solve the radiative transfer equation using the Feautrier method with 100 angular points and 3500 wavelength points. We include isotropic scattering except for Rayleigh scattering which can be added to the Feautrier method via a modified source function (Chandrasekhar 1960).

\subsection{Opacities}

We get the optical constants of $\mathrm{MgSiO}_{3}$ (enstatite) from Dorschner et al. (1995), of $\mathrm{Al}_{2} \mathrm{O}_{3}$ (corundum) from Koike et al. (1995) and Begemann et al. (1997), and of Fe (iron) from Ordal (1985) and Johnson \& Christy (1973). In all cases the optical constants were extrapolated below $0.2 \mu \mathrm{m}$. The condensate opacities (absorption and scattering) were computed using Mie theory for spherical particles with a version of the code from Bohren \& Huffman (1983). The condensate opacities dominate over gaseous Rayleigh scattering.

For $\mathrm{H}_{2} \mathrm{O}$, which is the dominant infrared absorber, we use the straight means opacities (Ludwig 1971). $\mathrm{TiO}$ is only present very deep in the atmosphere for the hottest models; we use straight means opacities from Collins \& Fay (1974). Even if the features do not appear in the atmosphere, the opacity contributes to the temperature-pressure structure. We also include $\mathrm{H}_{2}-\mathrm{H}_{2}$ and $\mathrm{H}_{2}$-He collision-induced opacities from Borysow et al. (1997), and Rayleigh scattering by $\mathrm{H}_{2}$ and $\mathrm{He}$ from Mathisen (1984). $\mathrm{CH}_{4}$ opacities are taken from the GEISA database (Husson et al. 1994) which is incomplete for the high temperatures of 
the CEGPs. Unfortunately the only existing optical $\mathrm{CH}_{4}$ opacities are coefficients derived from Jupiter (Karkoschka 1994), and we do not include them.

The alkali metals, noteably Na I and K I, are very important opacity sources in brown dwarf spectra (Tsuji et al. 1999; Burrows, Marley, \& Sharp 2000). The alkali metals' (Na, K, Li, Cs) oscillator strengths and energy levels were taken from Radzig \& Smirnov (1985). We only include the low-lying resonance lines which may have large absorption troughs in the optical. We compute line broadening using a Voigt profile with $\mathrm{H}_{2}$ and He broadening, and Doppler broadening.

Many better line lists exist, and other opacities that are present in L dwarf spectra may also appear in the CEGPs, but they are not necessary for a first approximation of light curves. We plan to include them in future work. While the opacities are necessary for a self-consistent solution of the atmosphere profile, the reflected spectra are not sensitive to small details in the infrared spectra.

\subsection{Condensates}

The atmospheric structure and emergent spectra of our "dusty" models are highly dependent on condensates, as first noted for brown dwarf models in Lunine et al. (1989), and subsequently by other modelers (e.g. Tsuji et al. 1996). The CEGPs have an extra sensitivity to condensates because the strong irradiation will heat up the upper atmosphere according to the condensate amount and absorptivity (see Figure (1). Also, because the incoming radiation is strongly peaked in the optical, in contrast to isolated brown dwarfs which have little optical emission, the condensates will cause strong reflection or absorption in the optical.

We consider four different sizes of condensates, based on the Solar System planets. The cases are intended to explore the expected size range, in part because the cloud theories are limited and may be in enough error that such assumptions are just as good. The particle sizes considered are mean radius $\bar{r}=$ $0.01 \mu \mathrm{m}, 0.1 \mu \mathrm{m}, 1 \mu \mathrm{m}$, and $10 \mu \mathrm{m}$. All have gaussian size distributions with a standard deviation of 0.1 times mean particle radius. This choice is narrow enough to attribute specific effects to a given particle size, but wide enough to prevent interference effects. Cloud particles in Venus have $r=0.85-1.15 \mu \mathrm{m}$, and a haze layer above that has particles with $r=0.2 \mu \mathrm{m}$ (Knibbe et al. 1997). The clouds on Jupiter range from an upper haze layer with $r=0.5 \mu \mathrm{m}$, and lower cloud decks with $r=0.75 \mu \mathrm{m}$, and $r=0.45-50 \mu \mathrm{m}$ (Taylor \& Irwin 1999). We assume that particles are distributed homogenously horizontally and vertically from the cloud base. The limitations of this assumption are discussed in $\$ 4.5$

For the light curve calculation with the Monte Carlo scattering code we compute the scattering matrix elements (Van de Hulst 1957) from Mie theory, which describe the anisotropic scattering phase function and the polarization.

\subsection{Monte Carlo method for scattering}

We use the atmosphere structure generated in the Seager \& Sasselov code and then compute the light curves and polarization curves using a Monte Carlo scattering code. In principle it is possible to compute the light curves with a model atmosphere code, but it is much more accurate to use the Monte Carlo code since it can deal with anisotropic scattering, the spherical geometry of the planet, and can easily compute all viewing angles - inclinations and phases — from one run. 
The basic principle of the Monte Carlo scattering method is that photon paths and interactions are simulated by sampling randomly from the various probability distribution functions that determine the interaction lengths, scattering angles, and absorption rates. Incoming photons at a given frequency travel into the atmosphere (to a location sampled from a probability distribution function), and scatter using random numbers to sample from probabilistic interaction laws. At each scatter, the photon's polarization and direction changes according to the phase function. Photons are followed until they are absorbed (they can no longer contribute to the reflected light), or until they exit the sphere. On exit, the photons are binned into direction and location; the result is flux and polarization as a function of phase and inclination.

The code we use was adapted from several previous codes described in the literature (Whitney 1991; Whitney \& Hartmann 1992, 1993; Code \& Whitney 1995; Whitney et al. 1999). Improvements from previous versions include exact sampling of the scattering phase function for any grain composition, arbitrary atmospheric density profiles, and inclusion of arbitrary opacity sources. Phase functions of $\mathrm{MgSiO}_{3}, \mathrm{Al}_{2} \mathrm{O}_{3}$, and $\mathrm{Fe}$ are computed using Mie theory. Additional opacities include Rayleigh scattering by $\mathrm{H}_{2}$ and $\mathrm{He}$, and absorption by $\mathrm{H}_{2} \mathrm{O}, \mathrm{TiO}, \mathrm{H}_{2}-\mathrm{H}_{2}, \mathrm{H}_{2}-\mathrm{He}$, and $\mathrm{H}^{-}$. Once absorbed, the photons are considered destroyed - they contribute to the thermal pool and no longer can contribute to scattered light. The Monte Carlo code uses the atmospheric structure (density profiles) and opacities computed from the detailed plane-parallel radiative and convective equilibrium code of Seager \& Sasselov, and computes scattering from a spherical planet with such an atmospheric structure. (As long as the scale-height of the atmosphere is small the plane-parallel approximation is sufficient to determine atmospheric structure). At visual wavelengths, the contribution of thermal emission from the planet is essentially zero and the reflected light can be treated as a scattering problem, where the incident radiation comes from the nearby star, and absorbed flux is ignored. Because condensate scattering is coherent we follow only one wavelength at a time. Because the CEGPs are so close to their parent stars that plane-parallel irradiation may not be accurate, we use the correct angular distribution of $\tan ^{-1}\left(R_{*} / D\right)$ (see $\left.\S 4.3 .2\right)$.

The Monte Carlo scattering method is preferable over "traditional" radiative transfer techniques because it can treat complex geometries, and its probabilistic nature gives all viewing angles at once. In the traditional plane-parallel method one can only solve along the line of sight, and must use the same angles for the incoming radiation as for the outgoing radiation (i.e. the emergent spectra). For the plane-parallel atmosphere models, one model must be computed for each phase angle and for each inclination. Our particular model atmosphere only considers isotropic or Rayleigh scattering, but realistically anisotropic scattering is important (see $\$$ t.3.2). Polarization is complicated and unnecessary when solving the model atmosphere for hydrostatic equilibrium, and for radiative and convective equilibrium in the traditional method.

Because of the need to use very large numbers of photons $\left(10^{7}\right.$ to $\left.5 \times 10^{8}\right)$ in order to fully sample the probability distribution function space, the Monte Carlo method cannot solve the model atmosphere problem (it is slow for optically thick regimes), although progress is being made in this direction for radiative equilibrium but with only a few line opacities (Bjorkman \& Wood 2000).

\section{Results and Discussion}

As an example of a CEGP we use $51 \mathrm{Peg}$ b (Mayor \& Queloz 1995). There are many uncertainties

about the CEGPs, including mass, radius, gravity, composition, $\mathrm{T}_{\text {eff }}$, etc. We have chosen only one example out of a large range of parameter space: $M=0.47 M_{\mathrm{J}}, \log g(\mathrm{cgs})=3.2$, metallicity that of the parent star, 
and $R_{P}=1.2 R_{J}$. (Note that the CEGPs' radii depend on mass, heavy element enrichment and parent star heating. Evolutionary models show $R_{P}$ for a given CEGP could be larger than the known radius of HD 209458b $\left(R_{P}=1.4 R_{J}\right)$ or as small as $0.9 R_{J}$ (Guillot 1999)). The incident flux of 51 Peg A (G2V, $\mathrm{T}_{\text {eff }}=5750$, metallicity $[\mathrm{Fe} / \mathrm{H}]=+0.21$, and $\log g(\mathrm{cgs})=4.4$ (Gonzalez 1998)) was calculated from the model grids of Kurucz (1992).

\subsection{Atmospheric Structure and Emergent Spectra}

We leave the detailed discussion of spectra and irradiative effects on temperature and pressure profiles for a separate paper. However, because the condensate assumptions affect the temperature-pressure profile and hence emergent spectra, we discuss the general properties here. These models supersede those in Seager \& Sasselov (1998) since grain formation and grain opacities are considered, most noteably, TiO condenses out of the upper atmosphere.

\subsubsection{Theoretical Spectra: Main Characteristics}

Figure 2 shows a model of $51 \mathrm{Peg} \mathrm{b}$ with a homogeneous cloud of $\mathrm{MgSiO}_{3}$ particles with $\bar{r}=0.01 \mu \mathrm{m}$, with $T_{\text {eff }}=1170 \mathrm{~K}$. The effective temperature $T_{\text {eff }}$ refers to the thermal emission only. The most noticeable feature is the large optical flux, many orders of magnitude greater than a blackbody (dotted line) of the same $T_{\text {eff }}$. The CEGPs have negligible optical emission of their own, although the hottest ones at $T_{\text {eff }}=1600 \mathrm{~K}$ may have some emission $>7000 \AA$, due to high absorption in the infrared which forces flux blueward. The CEGP in this model has 2-3 orders of magnitude more reflected flux than an isolated planet of the same $T_{\text {eff }}$ has emitted flux. The CEGPs are at very different effective temperatures from their parent stars of $\sim 6000 \mathrm{~K}$, so they have almost no molecular or atomic spectral features in common. Thus the spectral features in the blue and UV, blueward of $\sim 5200 \AA$, are largely spectral copies of the stellar spectra. Spectral features may also be reflected at longer wavelengths where no absorbers are present, for example $\mathrm{H} \alpha$ at $6565 \AA$.

The reflected optical component of the spectrum in this model comes from reflection from a homogeneous cloud of solid grains of $\mathrm{MgSiO}_{3}$ with particles with $\bar{r}=0.01 \mu \mathrm{m}$. Rayleigh scattering from $\mathrm{H}_{2}$ and $\mathrm{He}$ is negligible compared to the highly efficient scattering condensate, but plays a role deep in the atmosphere. The visual geometric albedo in this model is 0.18 . The reflected stellar features between 4000 and $5200 \AA$ follow the slope of the scattering coefficient of $\mathrm{MgSiO}_{3}$. In our models the condensate absorption and scattering features, such as the well known $10 \mu \mathrm{m}$ feature in comet reflectance spectra, do not emerge in the CEGP spectra, since they occur where thermal emission of the planet is strongest.

The absorption line at $7670 \AA$ is the $\mathrm{K} \mathrm{I} 4^{2} p-4^{2} s$ resonance doublet. Its broad wings extend for several hundred $\AA$ and are responsible for the slope redward to $1 \mu \mathrm{m}$. This effect is the cause of the large optical continuum depression in T dwarf spectra. (Tsuji et al. 1999; Burrows et al. 1999). This extreme broadening of the K I resonance doublet is also seen in cool L dwarf spectra (e.g. Tinney et al. 1999). Such broad atomic absorption of this kind — wings of thousands of $\AA$ — is not seen in any stellar atmosphere and indeed came as quite a surprise in the L dwarf observations. The cause is twofold: 1) strong pressure broadening of a fairly abundant species; and 2) there are no other strong absorbers in that wavelength region. The extreme broadening is not as surprising if we consider, for example, that if the Sun had no other absorbers than Lyman $\alpha$ (at $1215 \AA$ ) the wings would be visible out to the infrared (R. Kurucz, 
private communication). Other alkali metal lines are visible in the sample spectrum shown in Figure 2: Na I resonance doublet at $5893.6 \AA\left(3^{2} p-3^{2} s\right)$, Cs I at $8945.9 \AA\left(6^{2} p_{1 / 2}-6^{2} s\right)$ and $8523.5 \AA\left(6^{2} p_{3 / 2}-6^{2} s\right)$, Li I at $6709.7 \AA\left(4^{2} p-4^{2} s\right)$. With very low ionization potentials - between 3.89 and $5.39 \mathrm{eV}$ - the alkali metals are in neutral atomic form for much of the temperature-pressure regime in the CEGP atmosphere, although they do coexist with the gaseous metal chlorides and fluorides in the very upper atmospheres. The alkali metals are ionized in stars, and form alkali metal chloride solids in cooler planets such as Jupiter. Rb atomic lines should also be present but are not included in our model atmosphere. In principle Rayleigh scattering from Na I and K I could contribute a small amount to the scattering (Dalgarno 1968), but would only become important in condensate-free atmospheres.

The water bands are the most prominent absorption features in the infrared, with broad absorption troughs at 1.15, 1.4, 1.9, and $2.7 \mu \mathrm{m}$. Because the depth of the troughs is related to the temperature gradient, the spectral shape is expected to change depending on the amount of upper atmosphere heating, and to be different for irradiated planet atmospheres compared to isolated planet atmospheres. The infrared flux is thermal emission from absorbed and reradiated heat. Condensate absorption and scattering affects this wavelength region as well, as described in the next subsection.

The absorption trough at $3.3 \mu \mathrm{m}$ is $\mathrm{CH}_{4}$, and more minor methane features are apparent at 1.6 and $2.3 \mu \mathrm{m}$. The methane lines are strong for this particular model. However as described in the next subsection (4.1.2) the presence of methane at all is very sensitive to the amount of heating in the upper atmosphere.

\subsubsection{Effects of Condensates on Spectra}

The CEGP spectra are extremely dependent on the type, size, and amount of condensates in the planetary atmosphere, and Figure 2 represents only one specific model. Indeed it is impossible to predict the spectra, albedo, or light curve without referring to a specific condensate mix and size distribution. Figure 3 compares different low-resolution spectra at $\alpha=0$ of a subset of condensate cases considered in this paper. The curves are spectra from the following condensate assumptions: the solid line is a model with $\mathrm{MgSiO}_{3}$ particles of mean radius $\bar{r}=0.01 \mu \mathrm{m}$ (shown in Figure 2), the dotted line is a model with a $\mathrm{MgSiO}_{3}-\mathrm{Fe}-\mathrm{Al}_{2} \mathrm{O}_{3}$ mix of particles of $\bar{r}=0.01 \mu \mathrm{m}$, the dashed line is a model with a $\mathrm{MgSiO}_{3}-\mathrm{Fe}-\mathrm{Al}_{2} \mathrm{O}_{3}$ mix of particles of $\bar{r}=0.1 \mu \mathrm{m}$, and the dot-dashed line is a model with a $\mathrm{MgSiO}_{3}-\mathrm{Fe}-\mathrm{Al}_{2} \mathrm{O}_{3}$ mix of particles of $\bar{r}=10 \mu \mathrm{m}$.

The dotted curve is the most absorptive case which resembles a blackbody of $T_{\text {eff }}$ close to $T_{\text {eq }}$. Reflected features (not visible) appear at a very low magnitude. The most noticeable feature in the dashed curve is the broad dip between approximately $3000-10,000 \AA$. The cause is Rayleigh scattering from the condensates, which in this wavelength region have $\bar{r} \ll \lambda$. The slope is $\sim \lambda^{-4}$, but displaced compared to gaseous Rayleigh scattering since the Rayleigh scattering criterion is valid in a region of longer wavelength. The reflected spectral features are still visible on this Rayleigh scattering slope, in between the planetary atomic absorption lines. The K I and Na I lines are visible, but the extreme broadening shown in the solid line is not present because scattering and absorption high in the atmosphere means the deep atmosphere where the pressure broadening occurs is not sampled.

The most noticeable difference in the dot-dashed curve compared to the other spectra in Figure 3 is the presence of weak $\mathrm{TiO}$ features in the optical. Condensates with $\bar{r}=10 \mu \mathrm{m}$ have more scattering relative to absorption, and less absorption overall, right across the wavelength range. In this case, incoming radiation 
penetrates deep into the atmosphere, heating the atmosphere over a large depth to temperatures where enough $\mathrm{TiO}$ is present to produce weak absorption features.

$\mathrm{CH}_{4}$ is an excellent temperature diagnostic for the CEGPs' upper atmospheres. The strong $\mathrm{CH}_{4} 3.3$ $\mu \mathrm{m}$ band, and weaker $\mathrm{CH}_{4}$ features at 1.6 and $2.3 \mu \mathrm{m}$ are present only in the coolest, least absorptive model (solid line). The $\mathrm{H}_{2} \mathrm{O}$ bands also differ among the different models. They are much shallower for the atmospheres with absorptive condensates, due to absorption of incoming light by the condensates.

\subsubsection{Temperature-Pressure Profiles}

The temperature-pressure profiles (which are the basis for the emergent spectra) also vary depending on the type and size distribution of condensates in the planet atmosphere. Figure 0 shows the temperaturepressure profiles of the four models shown in Figure 3, together with the equilibrium condensation curves. In contrast to the $\mathrm{T}$ dwarfs which do not need clouds to be modeled, the irradiated CEGPs have heated upper atmospheres that bring the temperature closer to the equilibrium condensation curves so are more likely to have clouds near the top of the atmosphere. In Figure the model with particle mix with $\bar{r}=0.1 \mu \mathrm{m}$ (dashed line) is highly absorbing and results in a temperature inversion in the upper atmosphere layers. The model with cloud with particles with $\bar{r}=10 \mu \mathrm{m}$ is much less absorbing and results in a cooler temperature in the upper atmosphere layers. A highly reflective model (solid line) shows that much less heating occurs when molecules such as $\mathrm{H}_{2} \mathrm{O}$ are the primary absorbers. With the clouds at low pressure, at $10^{3}-10^{4}$ dyne $\mathrm{cm}^{-2}$, the equilibrium condensation curves for $\mathrm{MgSiO}_{3}$ and $\mathrm{Fe}$ are close together so a cloud mix of both particles could exist. Even if the uppermost cloud dominates the reflected light curve and spectra, the heating from lower cloud layers such as $\mathrm{Al}_{2} \mathrm{O}_{3}$ are important and do alter the temperature-pressure profile. The $T_{\text {eff }} \mathrm{s}$ of these models range from $1170 \mathrm{~K}$ to $1270 \mathrm{~K}$.

Another interesting consequence of the irradiative heating, evident from Figure 1 , is the proximity of the temperature-pressure profiles to the $\mathrm{CO} / \mathrm{CH}_{4}$ equilibrium curve. As noted in Goukenleuque et al. (1999) $\mathrm{CO}$ is expected to dominate over $\mathrm{CH}_{4}$, but $\mathrm{CH}_{4}$ is abundant enough to produce absorption bands. We have found that the strength of the $\mathrm{CH}_{4}$ features is sensitive to the upper atmosphere temperature which is in turn dependent on the amount of irradiative heating. Thus the $\mathrm{CH}_{4}$ bands are a good temperature diagnostic. (The $\mathrm{CH}_{4}$ bands are also useful, but less sensitive, as a pressure diagnostic (Seager 1999)).

Alternate approaches to modeling the temperature-pressure profiles have been taken. Marley et al. (1999) consider the temperature-pressure profile of an isolated object of the same effective temperature. Sudarsky et al. (1999) use ad hoc modified isolated temperature-pressure profiles (based on the temperaturepressure profiles in Seager \& Sasselov 1998), to simulate heating, instead of computing irradiative heating. As a result, the temperature gradient is much steeper because most of the heat comes from the bottom of the atmosphere. These models have clouds at the 1 bar level, near the bottom of the atmosphere. One consequence of this assumption is the strength of the K I and Na I absorption. Because of the clear atmosphere down to the 1 bar level, the K I and $\mathrm{Na}$ I resonance lines are extremely pressure broadened and absorb essentially all incoming optical radiation redward of $500 \mathrm{~nm}$. This is in contrast to the spectra shown in Figure 3 where the K I and Na I resonance lines are relatively narrow - the deep pressure zones where the broad line wings are formed are not sampled.

Although our approach is to compute the temperature-pressure profiles and reflected light in a consistent manner, we emphasize that in general there are many uncertainties in current CEGP models including photochemistry, cloud assumptions, and heat redistribution by winds. More specific to our models 
is the internal heat assumptions. For numerical reasons we must assume a lower boundary condition to our atmosphere in the form of a net flux coming from the planet interior. The assumption we have made is a net flux of approximately $1 / 10$ th of the absorbed flux. This may be too high (T. Guillot, private communication), and using a much lower value would produce a more isothermal atmosphere at the highest pressures in our models. More work is needed to understand the 3D heating redistribution in CEGPs. Importantly, the lower boundary condition has little effect on the upper atmospheric temperature and the reflected light curves.

\subsection{Condensates and the Scattering Asymmetry Parameter}

The shapes of the CEGP reflected light curves depend on the absorptivity and directional scattering probability of the condensates. Figure shows the scattering asymmetry parameter and the single scattering albedo at $5500 \AA$ for the three condensates considered as a function of particle size. The scattering asymmetry parameter $g$ is defined by

$$
g=<\cos \theta_{S}>=\int_{4 \pi} \cos \theta_{S} P^{11} \frac{d \Omega}{4 \pi},
$$

where $\theta_{S}$ is the scattering angle and $P^{11}$ is the phase function (see e.g. Van de Hulst 1957). The scattering phase function is the directional scattering probability of condensates (see Figures 9 and 13), and should not be confused with the planetary phase function introduced in $\delta$ 2 The scattering asymmetry parameter $g$ varies from -1 to 1 and is 0 for isotropic scattering. The higher $g$ is, the more forward throwing the particle. The curves for $g$ and $\tilde{\omega}$ in Figure 5 can predict, or help interpret, the light curves. Small particles compared to wavelength scatter as Rayleigh scattering; $g=0$ in this case, where the forward and backward scattering average out. This is seen for particles with $r=0.01 \mu \mathrm{m}$ (along the $y$ axis). In addition, $\mathrm{Al}_{2} \mathrm{O}_{3}$ and $\mathrm{Fe}$ have $\tilde{w}=0$ for $r=0.01 \mu \mathrm{m}$, so for these small particles absorption dominates over all scattering and the light curves will show little variation. For particles with $r=0.1 \mu \mathrm{m}$ more scattering will occur than for $r=0.01 \mu \mathrm{m} ; g=0.2$ and $\tilde{w}$ is high. In this case, scattering is not too forward throwing, and the probability of scattering over absorption is high. For particles with $r=10 \mu \mathrm{m}$, both $g$ and $\tilde{w}$ are high. High $g$ means the particle will scatter light preferentially in the forward direction. Coupled with high $\tilde{w}$, the photons will multiply scatter forward into the planet resulting in little reflected light. These effects will be partially borne out in the light curves shown in the next section.

The single scattering albedos and the asymmetry parameter curves are generally similar for large $r$ for the three particles considered because the parameters - indeed light scattering in general — are determined largely by the particle size compared to the wavelength of light. The curves are different from each other because of the different nature of the particles, specifically the real and complex indicies of refraction. At $5500 \AA, \mathrm{Fe}$ has a very high complex index of refraction, while that of $\mathrm{MgSiO}_{3}$ is essentially zero. The variations for a given curve, notably at $r=0.5 \mu \mathrm{m}$, are interference effects between diffracted light rays and rays that refract twice through the particle. This effect gets damped out for absorptive particles (e.g. Fe). For a concise discussion of asymmetry parameters and phase functions see Hansen \& Travis (1974).

Although we have chosen the dominant solids expected at the relevant temperatures and pressures from equilibrium calculations, it is certainly possible that nature has provided CEGPs with a different condensate size distribution, and different condensate particles and shapes than the ones used here. This will be investigated in future work. 


\subsection{Visual Photometric Light Curves and Polarization}

In the next few subsections we present the photometric light curves and fractional polarization curves. The results from our Monte Carlo scattering code give a planet-star flux ratio, and we use equation (4) to convert to variation in $\Delta m$, but where $\epsilon \phi(\Theta)$ is the planet-star flux ratio ( $\Theta$ is defined below). The results from the Monte Carlo scattering code also give the percent polarization, Pol, and we convert this to

fractional polarization of the system as described in $\$$, by $P_{\text {frac }}=\epsilon \phi(\Theta) P o l$, with the flux ratio in place of $\epsilon \phi(\Theta)$.

Figures 6a, 7a, Ba, and 11a show the $5500 \AA$ A light curves with orbital angle $\Theta$ for the four cases of particles with mean radius $\bar{r}=0.01,0.1,1$, and $10 \mu \mathrm{m}$. Here we use orbital angle instead of orbital phase used for radial velocity measurements because an angular variable is more convenient for the analysis. We define $\Theta$ as the angle in the orbital plane of the planet and star. With this definition, an orbital angle of $0^{\circ}$ occurs when the planet is farthest from Earth, and an orbital angle of $180^{\circ}$ occurs when the planet is between Earth and the star. In addition, for $i=90^{\circ}$ orbital angle and phase angle are equivalent, and orbital angle $0^{\circ}$ corresponds to orbital phase of $3 / 4$ (used in radial velocity measurements). In each figure the first curve is for inclination $i=90^{\circ}$, which for all CEGPs except HD $209458 \mathrm{~b}$, has already been excluded by transit non-detections. The other curves are for $i=82^{\circ}, i=66^{\circ}, i=48^{\circ}$, and $i=21^{\circ}$, none of which can be excluded by transit non-detections. The $y$ axis scale differs among the different figures. Transits occur only for $i \geq \theta_{T}\left(\theta_{T}=83.3^{\circ}\right.$ for $51 \mathrm{Peg} \mathrm{b}$ with $R_{*}=1.16 R_{\odot}, R_{P}=1.2 R_{J}$, and $\left.D=0.051 \mathrm{AU}\right)$, and within $\Theta=180^{\circ} \pm\left(90-\theta_{T}\right)$. They are barely visible on these figures, since transits darken rather than brighten the light of the system. In addition, the transit light curves are on the order of millimagnitudes, $\sim 2$ orders of magnitude greater than the reflected light effect. Nevertheless the start of the drop in the light curve for $i=90^{\circ}$ is shown at $\Theta=173.7^{\circ}$ and $\Theta=186.3^{\circ}$ at first and fourth contact respectively. Similarly, for $i=90^{\circ}$ - and only for $i>\theta_{T}$ - the reflected planetary light is not visible as the planet goes behind the star at $\Theta=360^{\circ}-\left(90^{\circ}-\theta_{T}\right)$, and reemerges at $\Theta=\left(90^{\circ}-\theta_{T}\right)$; that area is shaded in on the figures.

Figures [6 $\mathrm{b}, 7 \mathrm{~b}, 8 \mathrm{~b}$, and 11 $\mathrm{b}$ show the fractional polarization with orbital angle for the four cases of mean particle radius $0.01,0.1,1$, and $10 \mu \mathrm{m}$. Inactive solar-type stars are very weakly polarized, on the order of a few $\times 10^{-2}$ percent, so we treat the incoming light as unpolarized. We have plotted the fractional linear polarization of the system as described in $\S 2$, so that the polarization is modulated by the amount of scattered light, which peaks at an orbital angle of zero. Circular polarization is a secondary effect, and smaller than the errors in our scattering simulation.

\subsubsection{Particles with $\bar{r}=0.01 \mu m$}

Figure 6a shows the light curve for particles with $\bar{r}=0.01 \mu \mathrm{m}$, the case of very small particle size compared to wavelength. The amount of scattered light is tiny due to the high absorptivity of $\mathrm{Fe}$ and $\mathrm{Al}_{2} \mathrm{O}_{3}$, as described in $\$ 4.2$ That small particles obey Rayleigh scattering can be seen from the smooth, Rayleigh-like shape of the light curve. Rayleigh scattering produces more backscattering and results in a slightly different light curve from isotropic scattering. The Rayleigh scattering phase function $P_{R a y}=3 / 4\left(1+\cos ^{2} \theta_{S}\right)$ (where $\theta_{S}$ is the scattering angle), whereas for purely isotropic scattering $P_{i s o}=1$. At $\Theta=0^{\circ}$ and $i=90^{\circ}$, $P_{\text {Ray }}=1.5$, compared to $P_{i s o}=1$, and at $\Theta=90^{\circ}$ and $i=90^{\circ}, P_{\text {Ray }}=0.75$ which is smaller than $P_{i s o}=1$.

Rayleigh scattered light is maximally polarized at a scattering angle of $90^{\circ}$. Figure $6 \mathrm{~b}$ shows the fractional polarization of the CEGP system (described in $\$_{2}$ ), i.e. the ratio of polarized light to total white light of the star + planet. Because absorption is so high for this case, photons that exit the sphere have 
singly scattered, and the scattered light is $100 \%$ polarized. Even so, the fractional polarization is tiny because the amount of scattered light is very small compared to the unpolarized stellar flux. Figure $6 \mathrm{~b}$ also shows that different inclinations have the same polarization peaks, but with smaller amplitudes. Fractional polarization at $i=21^{\circ}$ is noisier than at other inclinations, since less radiation scatters into these phase angles, as indicated by the light curve in Figure ba.

We also ran simulations with $\mathrm{MgSiO}_{3}$ particles as the only condensate present in the planetary atmosphere (not shown in the figures), to investigate the lightcurves without the highly absorbing Fe and $\mathrm{Al}_{2} \mathrm{O}_{3}$ condensates (see Table 2). For $\bar{r}=0.01 \mu \mathrm{m}$ at $i=90^{\circ}$, the light curve peaks at $25 \mu \mathrm{mag}$, which corresponds to a geometric albedo $p=0.18$. Although $\tilde{\omega}$ is relatively high for this case, multiple scattering makes the resulting geometric albedo much smaller than for single scattering because it gives more chance for absorption. For the case where only $\mathrm{MgSiO}_{3}$ with particles of $\bar{r}=0.01 \mu \mathrm{m}$ is present, the polarization fraction peaks at $5.5 \times 10^{-6}$, which is almost 2 orders of magnitude higher than the $\mathrm{MgSiO}_{3}-\mathrm{Fe}-\mathrm{Al}_{2} \mathrm{O}_{3}$ mix. However, it is still below current detectability limits.

\subsubsection{Particles with $\bar{r}=0.1 \mu m$}

The light curves for particles with $\bar{r}=0.1 \mu \mathrm{m}$, shown in Figure $7 \mathrm{a}$, are similar to those for $\bar{r}=0.01 \mu \mathrm{m}$, but have a much larger amplitude. This can also be seen from Figure 5 which shows that for visual wavelengths the single scattering albedos are higher than those for $\bar{r}=0.01 \mu \mathrm{m}$, and the scattering asymmetry parameter is only 0.2 , which is reasonably isotropic.

Polarization, shown in Figure $7 \mathrm{~b}$, is also similar to the $\bar{r}=0.01 \mu \mathrm{m}$ case, with a much larger amplitude. Because the particles are still somewhat small compared to the wavelength of light, the scattering is largely Rayleigh scattering and the peaks are similar to those in Figure 6 b. However, not shown is that the scattered light is $55 \%$ polarized.

For this case of particles with $\bar{r}=0.1 \mu \mathrm{m}, \tilde{\omega}$ of Fe dominates. As seen from Figure 0 , if $\tilde{\omega}$ from $\mathrm{MgSiO}_{3}$ or $\mathrm{Al}_{2} \mathrm{O}_{3}$ dominates instead, the light curve will have a higher amplitude. For example, for a model with pure $\mathrm{MgSiO}_{3}$ clouds, the $i=90^{\circ}$ light curve peaks at $95 \mu$ mag, which corresponds to a geometric albedo $p=0.69$, and the fractional polarization peaks at $8.6 \times 10^{-5}$. The $i=21^{\circ}$ light curve peaks at $42 \mu$ mag. This case has the highest reflectivity of all of our models.

\subsubsection{Particles with $\bar{r}=1 \mu m$}

For particles with $\bar{r}=1 \mu \mathrm{m}$, which are larger than visual wavelengths, the light curve shown in Figure 8 a shows effects both from forward throwing and from a narrowly peaked backscattering function. Figure 9 a shows the phase function for the three different condensates, plotted with scattering angle $\theta_{S}$. Although the phase function represents single scattering, it can be used to interpret the light curve which arises from multiple scattering. The narrow backscattering (at $\theta_{S}=180^{\circ}$ ) is responsible for the narrow peak in the light curve: there is a high probability of backscattering but only for a narrow angular range. The high probability for forward throwing means that photons are likely to be forward scattered into the atmosphere where they will be absorbed; this is the cause for the otherwise reduced light curve (in the "wings") compared to the Rayleigh-shaped light curves in Figures 6a and 7a.

We also plot the phase function as a polar diagram in Figure 9 $\mathrm{b}$, where the light is incoming from 
the left, and the condensate particle is marked at the origin. Figures $9 \mathrm{a}$ and $9 \mathrm{~b}$ also show that the three condensates have different amounts of backscattering, indeed slightly different phase functions overall. In Figure 10a we plot the three light curves from each of the condensates, considering that each condensate is the only one present in the atmosphere. This shows that the $\tilde{\omega}$ of $\mathrm{MgSiO}_{3}$ dominates, as compared to particles with $\bar{r}=0.01 \mu \mathrm{m}$ where Fe dominates, and also that each condensate has unique properties. Both Fe and $\mathrm{Al}_{2} \mathrm{O}_{3}$ have very forward throwing phase functions without a strong backward peak; the result is that incoming light is forward scattered into the atmosphere where it is not likely to contribute to reflected light. As a result, their light curve amplitudes are much smaller than $\mathrm{MgSiO}_{3}$ 's; $\mathrm{Al}_{2} \mathrm{O}_{3}$ alone would not even be detectable by the planned microsatellites. The same comparison for particles with $\bar{r}=0.01 \mu \mathrm{m}$ and $\bar{r}=0.1 \mu \mathrm{m}$, where the phase functions and light curves are more isotropic, reveals that the main effect of each condensate is mostly a change in magnitude. This is because the particles are smaller than the wavelength of light, and to first order light is Rayleigh scattered.

The phase function of $\mathrm{MgSiO}_{3}$, the solid curve in Figure $9 \mathrm{a}$, is typical of those for spheres of size parameter $x \geq 1$, with complex index of refraction $n_{i} \sim 0$ (see Hansen \& Travis 1974), where $x=2 \pi r / \lambda$. The forward throwing is caused by diffraction of light rays around the particle and depends on the geometrical cross-section of the particle, and so would also occur for non-spherical particles. The backward peak, known as the "glory", is specific to spherical particles and is related to interfering surface waves on the particle sphere. For absorbing particles (with high $n_{i}$, such as $\mathrm{Fe}$ ), this effect is damped out, as seen from the Fe phase function in Figure 9 a, which shows no rise towards $\theta_{S}=180^{\circ}$. For randomly oriented axi-symmetric spheroids, the backward peak would not be as severe (Mishchenko et al. 1997). The phase function of randomly oriented axi-symmetric spheroids depends on the distribution of both particle axis sizes and particle orientation. To estimate the light curve from a reduced backscattering peak, we assume all of the condensates scatter like $\mathrm{Fe}$, and in another case all like $\mathrm{Al}_{2} \mathrm{O}_{3}$. In other words, we use the same total opacity of the $\mathrm{MgSiO}_{3}-\mathrm{Fe}-\mathrm{Al}_{2} \mathrm{O}_{3}$ mix. The resulting light curves are shown in Figure 10 b. Although their peaks are much lower than the strong backscattering case, with the exception of $\mathrm{Al}_{2} \mathrm{O}_{3}$ this variation is still detectable by the upcoming microsatellite missions.

Because the CEGPs are very close to their parent stars, light rays hitting the planet may not be well approximated as plane-parallel. We use the correct angular distribution of $\tan ^{-1}\left(R_{*} / D\right)$ ( $6^{\circ}$ for 51 $\mathrm{Peg} \mathrm{b})$. The main effect from using this angular distribution compared to plane-parallel rays is that the backscattering peak is reduced by $12 \%$, because the backscattering phase function peaks sharply at $180^{\circ}$. A more minor improvement is that the lower inclination light curves are a few percent lower. For isotropic or Rayleigh scattering we find no difference in the light curve from using either plane-parallel rays or the correct angular distribution of incoming radiation. Because $\tan ^{-1}\left(R_{*} / D\right)$ is such a small angle, isotropic irradiation, used in atmosphere codes that treat feedback from the star's own corona, is not accurate.

The polarized light curve shown in Figure $8 \mathrm{~b}$ is very different from the Rayleigh scattering polarization curves for $\bar{r}=0.01 \mu \mathrm{m}$ and $\bar{r}=0.1 \mu \mathrm{m}$. The polarization is more complex than Rayleigh scattering as the light rays reflect from and refract through the particles, and the scattered light rays interfere. In addition, the polarization from each condensate is different, since polarization depends in part on the index of refraction, which is very different for each of the three condensates in this study. The peak of the polarization has a similar peak to the light curve, since fractional polarization is plotted which follows the scattered light. Polarization of the scattered light alone shows a smaller central peak and additional smaller peaks at $10^{\circ}$ and at $70^{\circ}$ for $i=90^{\circ}$. 


\subsubsection{Particles with $\bar{r}=10 \mu \mathrm{m}$}

Light curves from particles with $\bar{r}=10 \mu \mathrm{m}$, shown in Figure 11a, are similar to the light curves from $\bar{r}=1 \mu \mathrm{m}$ particles, but with a more pronounced effect from strong forward throwing and an even more narrowly peaked backscattering probability. Outside of the backscattering peak, the light curve is much smaller due to the forward throwing effects discussed above. For $i=90^{\circ}$ and $\Theta=160^{\circ}$, there is a rise in the light curve just before the transit. This is from light that enters the atmosphere near the limb, and scatters through the top of the atmosphere due to the high forward throwing nature of the large particles. For particles with $\bar{r}=10 \mu \mathrm{m}$, the polarization is very similar to the $\bar{r}=1 \mu \mathrm{m}$ case, but with a greater peak from the greater amount of backscattering, and an otherwise lower amplitude from the higher forward throwing.

\subsection{U, B, V, R Photometric Light Curves and Polarization}

In this section we compare the light curves and polarization at the $\mathrm{U}, \mathrm{B}, \mathrm{V}$, and $\mathrm{R}$ effective wavelengths $(\mathrm{U}=3650 \AA, \mathrm{B}=4400 \AA, \mathrm{V}=5500 \AA, \mathrm{R}=7000 \AA)$. The incoming stellar flux has many spectral features over the wavelength range of a band, but the same features are reflected by the planet (Figure 3); the light curve depends on the flux ratio and the features cancel out. However, for a second order calculation absorption by alkali metal line wings (in the planetary atmosphere) or the opacity variation from condensates within a color band may play a role.

In general the light curves are a function of the opacity and of the phase function. The opacity effects include density effects and the single scattering albedo. Photons travel into the atmosphere and encounter condensates (or atoms or molecules) which will scatter or absorb them. If the photons scatter, they will scatter according to the condensate phase function. A phase function that preferentially scatters photons into the forward direction will generate a very different light curve than a Rayleigh phase function, as shown in $\$ 4.3$ The phase function for given optical constants depends on the size parameter $x=2 \pi r / \lambda$, and the condensate index of refraction.

Figure $12 \mathrm{a}$ shows the light curves of the $51 \mathrm{Peg} \mathrm{b}$ system for particles with $\bar{r}=0.1 \mu \mathrm{m}$. The main difference between the colors is caused by the different size parameters: different wavelengths of light for a fixed particle size. For example, $x=0.90$ at $\mathrm{R}$ but $x=1.72$ at $\mathrm{U}$. The effects of this are seen in the light curves: $\mathrm{R}$ has a Rayleigh-scattering-like light curve compared to $\mathrm{U}$. The phase functions for the 4 colors for $\mathrm{Fe}$ are shown in Figure 13. As mentioned in \$4.3.2. Fe opacity dominates this case of particles with $\bar{r}=0.1 \mu \mathrm{m}$. In fact, the $\mathrm{U}$ light curve is close to the shape of the Fe-only light curve for $\bar{r}=1 \mu \mathrm{m}$ particles at $\mathrm{V}$ (dashed line in Figure (10a)). Because of the $x$ dependency of the phase functions, the different colors for a fixed size go through the same shapes as shown for the $\mathrm{V}$ light curves which describe fixed wavelength for a varying particle size. However, there are differences due to opacity variation with color.

Figure 12b shows the polarization fraction for $\bar{r}=0.1 \mu \mathrm{m}$. Effects from both phase function and opacity contribute. The polarization peak of each color is at a different angle, due to the different indicies of refraction of the particles at different colors. The difference in the polarization peaks is much greater than the difference in the light curve peaks between the colors. The polarization curves reflect the higher asymmetry in the scattering in $\mathrm{U}$ and the greater absorption at this wavelength. 


\subsection{Cloud Layers}

The light curves and polarization curves presented in this paper have been computed under the assumption that the clouds are not in layers, but that above a given equilibrium condensation curve all of the gas condenses into solids and the particles are suspended uniformly in the atmosphere.

There are two consequences of finite cloud layers. The first difference is that stratification will separate different condensates into different layers, where the light curve and flux signature will come largely from the cloud closest to the top of the atmosphere. In contrast to our models of vertically homogenous clouds, a cloud confined to one pressure scale height with the same solid mass fraction would have a higher optical depth. With a high optical depth the reflected light cloud signature is even more likely to resemble the uppermost cloud only than the situation of a homogenous mix.

The second difference is that the gaseous scatterers and absorbers above the cloud layer could play a role. The dominant gaseous scatterer is Rayleigh scattering by $\mathrm{H}_{2}$, and the dominant absorbers are alkali

metals, particularly K I (7670 $)$ ) and Na I (5894 $\AA)$. In our approximate models with cloud layers, the alkali metals are strong, but narrow. Because the irradiated temperature-pressure profiles cross the condensation boundaries at relatively low pressure, the strong pressure broadening of the alkali metals does not occur in our models.

With the choice of a finite cloud layer, an additional free parameter becomes the location of the cloud base. For example, in the case considered here the temperature-pressure curve could cross the Fe and $\mathrm{MgSiO}_{3}$ condensation curves at low $P$, where they overlap. In this case a $\mathrm{Fe}-\mathrm{MgSiO}_{3}$ mix will prevail

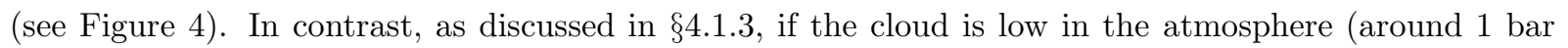
e.g. Sudarsky et al. 1999) the incoming radiation will be absorbed by broad lines of Na I and K I before reaching the scattering cloud, causing zero optical albedo redward of $500 \mathrm{~nm}$.

We have rudimentarily explored finite cloud layers where all clouds are 1 pressure scale height above their base at the equilibrium condensation curve. The main difference in reflected light curves from our vertically homogenous assumption is an increase in magnitude. The reason is that $\mathrm{MgSiO}_{3}$, which has the coolest condensation curve (shown in Figure 1 ), would be the top layer; $\mathrm{MgSiO}_{3}$ is both the most reflective (see $\tilde{\omega}$ in Figure 5) and has the largest backscattering probability of the three condensates considered here. The light curve shape changes little with the cloud layer models. In the case of small particles compared to wavelength $(\bar{r}=0.01 \mu \mathrm{m}$ for $5500 \AA)$, the shape of the phase function is Rayleigh-like for all three condensates. In the case for large particles compared to wavelength $(\bar{r}=10 \mu \mathrm{m}$ for $5500 \AA)$, $\tilde{\omega}$ of $\mathrm{MgSiO}_{3}$ already dominates in the three condensate mix for large particles. However, we caution that much more work needs to be done both in cloud models and particle size distribution. We emphasize the difficulty in predicting the reflected light curves due to the large parameter space of irradiative heating, cloud models, and particle type and size distribution. Thus these exploratory models should be considered as a useful interpretative tool rather than a predictive tool.

\subsection{Comparison with Observations}

\subsubsection{Spectral Separation}

Cameron et al. (1999) and Charbonneau et al. (1999) have given the first observational results for a CEGP atmosphere, $\tau$ Boo b. The results are marginally in conflict (see below); the first group claims a 
probable detection with a flux-ratio of $\epsilon=1.9 \times 10^{-4}$ and the second group a null result with upper limit of $\epsilon=5 \times 10^{-5}$. Although in this paper we are modeling $51 \mathrm{Peg} \mathrm{b}$, we can assume to first order that $\tau$ Boo $\mathrm{b}$ has a similar atmosphere. However, $\tau$ Boo b is hotter than $51 \mathrm{Peg} \mathrm{b}$ and may be heated above the condensation boundary of some grain species.

To first order such observations are extremely useful in addressing whether or not there are reflective clouds near the top of the atmosphere. For example, if the probable Cameron et al. result is confirmed, $\tau$ Boo b must have a very reflective cloud of particles fairly high in the planet atmosphere. The reason for this is the orbital inclination was measured to be $29^{\circ}$, meaning only small phase angles are observed during the planet's orbit and the planet must be very bright to be detectable at all. If the particles are spherical, they must also be smaller than the wavelength of light, because the strongly peaked wavefunctions of highly reflecting spherical particles (e.g. particles with $\bar{r}=10 \mu \mathrm{m}$ with light curve shown in Figure 11) generate a small light curve amplitude at low inclinations. Cameron et al. also find a wavelength dependence of the albedo. If confirmed this will reveal molecular absorbers in the atmosphere, but not cloud characteristics (which are grey at optical wavelengths).

The Charbonneau et. al result is inclination dependent, and provides useful constraints if the system is at high orbital inclination. Their upper limit of $p=0.3$ at high inclinations excludes atmospheres with extremely reflective clouds (such as pure $\mathrm{MgSiO}_{3}$ clouds of small particles.) Bright models with light curve shapes different from isotropic, e.g. those shown in Figures 5 and 6 (which have $p>0.3$ for high $i$ ) are not excluded, because of the phase function assumption (see below). Their upper limit is based on the assumption that the reflected planetary light is an exact copy of the stellar light for 4668 to $4987 \AA$. For lower inclinations $\left(i \approx 30^{\circ}\right)$, Charbonneau et al. give an upper limit of $1 \times 10^{-4}$. This does not provide a useful model constraint because at low inclinations only small phases of the planet are visible, and only a few extreme cases of highly reflective clouds can be excluded.

Beyond characterizing the very general cloud reflectance property, the spectral separation observations cannot constrain the particle type or size distribution. One reason is that the observations measure a combination of the geometric albedo and planet area: $p\left(R_{P} / D\right)^{2}$. The planet radius is not known, except for a transiting planet. Cameron et al. assume a Jupiter-like albedo of $p=0.55$ and derive $R_{P}=1.8 R_{J}$; Charbonneau et al. assume $R_{P}=1.2 R_{J}$ (based on evolutionary models from Guillot et al. 1996) and derive an upper limit for $p$.

A second difficulty in using the spectral separation results to constrain cloud details is the phase function assumption that goes into the results. The results given by both groups are expressed as a flux ratio or geometric albedo at opposition. Neither group observed near opposition ("full phase" for $i=90^{\circ}$ ), but instead extrapolated their results based on an assumed phase function. Cameron et al. used an empirically determined polynomial approximation to the phase function of Venus (Hilton 1992), which also resembles that of Jupiter (Hovenier \& Hage 1989). Charbonneau et al. used the Lambert sphere phase function, which derives from isotropic scattering and is approximately valid for Uranus and Venus. The differences in $p$ from using these different phase function assumptions is $20 \%$ and this may be one contribution to the conflicting observational results. For more details see Cameron et al. (2000) and Charbonneau \& Noyes (2000). The inherent uncertainty in the albedo measurement from both the phase function and the radius/albedo degeneracy prevents any serious constraint on atmosphere models. Nevertheless, for comparison Table 2 shows the geometric albedos of our 4 models at $4800 \AA$, roughly the center of both groups' wavelength range.

Results from this paper show that the light curve observations needed to constrain atmosphere models 
are those at different colors in narrow wavelength bands. The specific anisotropic scattering properties for a given particle size distribution and grain indicies of refraction will determine the light curve. The indicies of refraction are both wavelength- and particle type-dependent, which is why the color dependence is important. Opacity effects in a narrow wavelength range are less important because the condensates are generally grey in the optical.

\subsubsection{Ground-based Photometric Light Curves}

Observations by G. Henry (private communication) with ground-based automatic photometric telescopes can currently reach a precision of near $100 \mu \mathrm{mag}$ and could be as precise as $50 \mu \mathrm{mag}$ with a dedicated automatic photometric telescope. The precision is attainable with observations over many orbital periods because the phase effect is strictly repeating. With this limit, reflected light detections of high-orbital inclination systems should be possible, or at least useful constraints on the models. Furthermore, these photometric limits should allow confirmation of the Cameron et al. result. Their result of $\epsilon=1.9 \times 10^{-4}$, which using the polynomial approximation to Venus (Hilton 1992) for $i=29^{\circ}$ translates roughly into a flux-ratio of $9 \times 10^{-5}$ and an amplitude of $7 \times 10^{-5}$ which corresponds to $\approx 80 \mu \mathrm{mag}$.

\subsection{Other EGPs}

\subsubsection{Close-in EGPs}

Because $\epsilon \sim\left(R_{P} / D\right)^{2}$, the flux variation of other CEGPs can be estimated using the data in Table 1 , and multiplying the light curves by the $\left(D_{51 P e g} / D_{E G P}\right)^{2}$ ratio. However $\Delta m$ is a flux ratio and is not affected by the magnitude of the parent star, although magnitude is observationally important. This estimate also assumes that the radii of the planets are the same (cf. Guillot et al. 1996). This estimate, shown for a Lambert sphere in Figure 1, shows a variation of a factor of 2 between $D=0.042 \mathrm{AU}$ and $D=0.059 \mathrm{AU}$.

There are additional differences among the different CEGPs that affect scattering. One is from density. For example, $51 \mathrm{Peg} \mathrm{b}$ has almost an order of magnitude lower minimum mass than $\tau$ Boo $\mathrm{b}$ for the same planetary radius. A less dense atmosphere has a longer photon mean free path, which has two effects. One is more backscattered light. The second, which is minor, is more unscattered radiation passing through the limb, and less forward-scattered radiation traveling through the upper atmosphere. Coincidentally, some of the enhanced scattering effects gained from 51 Peg b's lower surface gravity atmosphere compared to $\tau$ Boo b are lost with the larger distance from the parent star. With a lower surface gravity, 51 Peg b's atmosphere is less dense (has a lower $P_{g}$ ) for the same Rosseland mean optical depth. Figure 14 shows the light curves for both $\tau$ Boo b and $51 \mathrm{Peg} \mathrm{b}$, for the three condensate mix of particles with $\bar{r}=10 \mu \mathrm{m}$, at $i=82^{\circ}$, which is not excluded by a transit non-detection. Also plotted in Figure 14 is $51 \mathrm{Peg}$ b's light curve at $D_{\tau \text { Boo }}$. Because of its lower surface gravity atmosphere, $51 \mathrm{Peg} \mathrm{b}$ shows effects from more scattering; a higher backscattering peak $\left(335^{\circ}<\theta_{S}<25^{\circ}\right)$, and more light scattered through the upper atmosphere $\left(\theta_{S}>170^{\circ}\right)$. The difference at $25^{\circ}<\theta_{S}<335^{\circ}$ is also due to the different atmosphere densities. Figure 14 shows that the observations would not be able to constrain the density; away from $\Theta=0^{\circ}$ the differences are very small, and near $\Theta=0^{\circ}$ the amplitude difference is degenerate with change in density, $\tilde{\omega}, R_{p}$, etc.

A planet with a larger radius than we have assumed, such as $R_{P}=1.40 \pm 0.17 R_{J}$ derived from the 
transit of HD 209458 b (Mazeh et al. 2000) would have a larger reflected light signal. The density effects described above would be a secondary effect.

Discrete cloud layers (not modeled here but discussed in \$4.5) could have an effect on the CEGPs. The bases of different cloud types may be at different depths, as on Jupiter, because thermodynamical equilibrium calculations predict condensation curves with different temperature and pressure dependencies for each species (see Figure 1). CEGPs that have a hotter parent star or a smaller $D$ will have different temperature-pressure structures than the cooler ones; they may be different enough that different cloud layers are more or less visible. For example, we find that for particles with $\bar{r}<1 \mu \mathrm{m}, \tau$ Boo b's atmosphere may be heated enough to have a temperature above that of $\mathrm{MgSiO}_{3}$ condensation.

\subsubsection{EGPs Beyond 0.05 AU}

Because we are investigating planets that could be detectable in reflected light in the near future, we focus on the CEGPs. However, for a very rough estimate of EGPs with $D>0.05 \mathrm{AU}$, the light and polarization curves in this paper can be scaled by $\left(D_{51 P e g} / D_{E G P}\right)^{2}$ (since $\left.\epsilon \sim 1 / D^{2}\right)$. This rough estimate ignores the cloud particles, which would be different than those in the CEGP atmospheres. An EGP such as $\rho^{1} \mathrm{Cnc}$, at $0.12 \mathrm{AU}$ from its parent star, is 2.35 times as far as $51 \mathrm{Peg} \mathrm{b}$ from its parent star. The maximum amplitude light curve in this paper $\left(60 \mu \mathrm{mag}\right.$ at $\left.i=90^{\circ}\right)$ would be only be $11 \mu$ mag at the distance of $\rho^{1}$ Cnc. This variation is barely detectable by the upcoming microsatellite missions. An EGP such as $\rho$ CrB at $D=0.23 \mathrm{AU}$ is 4.5 times farther from its parent star than $51 \mathrm{Peg} \mathrm{b}$. The maximum amplitude light curve at this distance is only $3 \mu \mathrm{mag}$. Such an EGP will not be photometrically detectable in the foreseeable future. Thus the CEGPs have the brightest prospects for detection.

\section{Summary and Conclusion}

We have presented photometric light curves and fractional polarization curves for $51 \mathrm{Peg} \mathrm{b}$ for 4 mean particle sizes, and discussed the differences with color and with other CEGPs. The light curves are very sensitive to condensate type and size distribution, hence observations will be able to distinguish between extreme scenarios. However, more detailed information such as the exact size distribution and particle type will be more difficult to extract.

The temperature-pressure profiles are also extremely dependent on the condensate assumptions. We have briefly discussed these, along with the emergent spectra. In contrast to $T$ dwarfs which have no observeable clouds, the CEGPs should have clouds closer to the top of their atmospheres because irradiation heats the upper atmosphere to temperatures closer to the equilibrium condensation curve (i.e. the cloud base). The condensates that may not contribute to reflected light because they are sequestered below the top cloud layer will still affect the temperature-pressure profile by heating the lower atmosphere. Thus observations of the light curves which should constrain the general cloud properties will help distinguish between atmosphere models.

The light curves may be very different from sine curves; their shape depends not just on the particle size and type, but also on $\tilde{\omega}$ and the atmospheric density. Inclinations other than the narrow angular range possible for transits are theoretically detectable for the CEGPs. Many cases of the light curves are detectable by upcoming space missions, and some of the largest amplitude cases (e.g. from pure $\mathrm{MgSiO}_{3}$ 
particles with $\bar{r}=0.1 \mu \mathrm{m}$ ) might be detectable from the ground in the near future. See Table 2, and

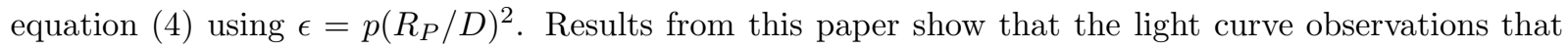
would best constrain atmosphere models are those at different colors in narrow wavelength bands.

Geometric albedos at $5500 \AA$ in this study for a cloud mix of particles of $\mathrm{MgSiO}_{3}, \mathrm{Fe}$, and $\mathrm{Al}_{2} \mathrm{O}_{3}$ range from $p=0.44$ for particles with $\bar{r}=10 \mu \mathrm{m}$ that have a strong backscattering peak, to $p=0.0013$ for particles with $\bar{r}=0.01 \mu \mathrm{m}$ which include highly absorbing Fe. Clouds of pure $\mathrm{MgSiO}_{3}$ are much more reflective for all particle sizes (see Table 2).

The polarization of the CEGP systems is not detectable with current techniques. Rayleigh scattering polarization peaks at orbital angle $90^{\circ}$, but with modulation of the reflected light the fractional polarization has an asymmetric peak at around $70^{\circ}$. Polarization from particles that are large compared to the wavelength will have more than one peak due to interference effects from different light ray paths through the particle.

Other CEGP systems in our study give similar light curves to $51 \mathrm{Peg} \mathrm{b}$, but effects from distance from the parent star and density are important. Planets farther than $0.1 \mathrm{AU}$ from their parent stars are too faint in reflected light to be detected photometrically in the foreseeable future.

We emphasize that there are many unknowns in the model atmospheres, and much room for improvement - mainly more realistic cloud modeling, heat redistribution by winds, photochemistry, non-spherical particles, and other types of condensates. These ingredients will result in different light curves than those shown in this paper. That the predictions are so varied means observations should be able to identify the gross cloud characteristics. In this sense the theory should be seen as an interpretive rather than a predictive tool. Observations by the upcoming satellites MOST, COROT, and MONS and ground-based work will help constrain the CEGP atmosphere models and at best will reveal the nature of their atmospheres directly.

We thank Kenny Wood for illuminating discussions on the Monte Carlo scattering method, Dave Charbonneau for many useful discussions, Jens Falkesgaard for many of the Gibbs Free energy fits. We also thank Mark Marley, Bob Noyes, Mike Wolff, Greg Henry, and Adam Burrows for useful discussions. We thank the referee Tristan Guillot for helpful comments that improved the paper. SS is supported by NSF grant PHY-9513835, BAW acknowledges support by NAG5-8587, and DDS acknowledges support from the Alfred P. Sloan Foundation. 
The Close-in Extrasolar Giant Planets

\begin{tabular}{lcccccl}
\hline \hline Star Name & Spectral Type & $\begin{array}{c}\mathrm{D} \\
(\mathrm{AU})\end{array}$ & $\begin{array}{c}M \sin i \\
\left(M_{J}\right)\end{array}$ & $\begin{array}{c}\mathrm{P} \\
(\text { days })\end{array}$ & $\begin{array}{c}T_{\mathrm{eq}}(1-A)^{-1 / 4} \\
(\mathrm{~K})\end{array}$ & Reference \\
\hline HD 187123 & G3V & 0.042 & 0.52 & 3.097 & 1400 & 1 \\
HD 75289 & G0V & 0.046 & 0.42 & 3.51 & 1600 & 2 \\
$\tau$ Boo & F7V & 0.0462 & 3.87 & 3.3128 & 1600 & 3 \\
HD 209458 & G0V & 0.0467 & 0.69 & 3.525 & 1500 & 4 \\
51 Peg & G2V & 0.051 & 0.47 & 4.2308 & 1300 & 5 \\
\hline
\end{tabular}

References. - (1) Butler et al. 1998; (2) Mayor et al. 1999; (3) Butler et al. 1997; (4) Mazeh et al. 2000; (5) Mayor \& Queloz 1995

Geometric Albedos

\begin{tabular}{lccc}
\hline $\begin{array}{l}\text { Mean Particle } \\
\text { Size }\end{array}$ & $\lambda=5500 \AA$ & $\begin{array}{c}\lambda=5500 \AA \\
\mathrm{MgSiO}_{3} \text { only }\end{array}$ & $\lambda=4800 \AA$ \\
\hline $0.01 \mu \mathrm{m}$ & 0.0013 & 0.18 & 0.0013 \\
$0.1 \mu \mathrm{m}$ & 0.18 & 0.69 & 0.14 \\
$1 \mu \mathrm{m}$ & 0.41 & 0.50 & 0.36 \\
$10 \mu \mathrm{m}$ & 0.44 & 0.55 & 0.4 \\
\hline
\end{tabular}

Note. - Geometric albedos for the models discussed in this paper (column 1), for pure $\mathrm{MgSiO}_{3}$ clouds which are highly reflective (column 2), and for the models in this paper at $\lambda=4800 \AA$ (third column), which corresponds to the Cameron et al. (1999) and Charbonneau et al. (1999) observations. 


\section{REFERENCES}

Allard, F., \& Hauschildt, P. H. 1995, ApJ, 445, 433

Akeson, R., \& Swain, M. 1999, in ASP Conf. Ser. From Giant Planets to Cool Stars, ed. C. Griffith \& M. Marley, in press

Baglin, A. 1998, in New Eyes to See Inside the Sun and Stars, ed. F-L. Deubner et al., p.301

Baglin, A. 2000, http://www.astrsp-mrs.fr/www/pagecorot.htm

Baliunas, S. A., Henry, G. W., Donahue, R. A., Fekel, F. C., \& Soon, W. H. 1997, ApJ, 474, L119

Begemann, B., Dorschner, J., Henning, Th., Mutschke, H., Guertler, J., \& Koempe, C. 1997, ApJ, 476, 1999

Bjorkman, J. E. \& Wood, K. 2000, in preparation

Bohren, C. F., \& Huffman, D. R. 1983, Absorption and Scattering of Light by Small Particles, (New York: John Wiley and Sons)

Bond, 1861, Proc. Amer. Academy of Arts and Sciences, N.S., 8, 232

Borysow, A., Jorgensen, U. G., \& Zheng, C. 1997 A\&A 324, 185

Burrows, A., Marley, M., \& Sharp, C. M. 2000, ApJ, in press, astro-ph/9908078

Burrows, A., Marley, M., Hubbard, W. B., Lunine, J. L., Guillot, T., Saumon, D., Freedman, R., Sudarsky, D., \& Sharp, C. 1997, ApJ, 491, 856

Burrows, A. \& Sharp, C. M. 1999, ApJ, 512, 843

Butler, R. P., Marcy, G. W., Vogt, S., \& Apps, K. 1998, PASP, 110, 1389

Butler, R. P., Marcy, G. W., Williams, E., Hauser, H., \& Shirts, P. 1997, ApJ, 483, 457

Cameron, A. C., Horne, K., Penny, A., \& James, D. 2000, in ASP Conf. Ser., Disks, Planetesimals, and Planets, ed. F. Garzon, C. Eiroa, D. de Winter, \& T. J. Mahoney, in preparation

Cameron, A. C., Horne, K., Penny, A., \& James, D. 1999, Nature, 402, 751

Chandrasekhar, S. 1960, Radiative Transfer, (New York: Dover)

Charbonneau, D., Brown, T. M., Latham, D. W., Mayor, M. 2000, ApJ, 529, 45L

Charbonneau, D. \& Noyes, R. W. 2000, in ASP Conf. Ser., Disks, Planetesimals, and Planets, ed. F. Garzon, C. Eiroa, D. de Winter, \& T. J. Mahoney, in preparation, astro-ph/0002489

Charbonneau, D., Noyes, R. W., Korzennik, S. G., Nisenson, P., Jha, S., Vogt, S. S., Kibrick, R. I. 1999, ApJ, 522, L145

Christensen-Dalsgaard, J. 2000, http://bigcat.obs.aau.dk/ hans/mons/

Chase, M. W. 1998, NIST-JANAF Thermochemical Tables, 4th ed. (Washington DC: American Chemical Society)

Code, A. D., \& Whitney, B. A. 1995, ApJ, 441, 400

Collins, J. G., \& Fay, T. D. 1974, JQRST, 14, 1259

Dalgarno, A. 1968, unclassified document Dept. of Defense, AD297883

Dorschner, J., Begemann, B., Henning, T., Jaeger, C., Mutschke, H. 1995, A\&A, 300, 503

Eriksson, G. 1971 Acta. Chem. Scand., 25, 2651

Fegley, B., \& Lodders, K. 1996, ApJ, 472, L37 
Gonzalez, G. 1998, A\&A, 334, 221

Goukenleuque, C., Bezard, B. \& Lellouch, E. 1999, in ASP Conf. Ser. From Giant Planets to Cool Stars, ed. C. Griffith \& M. Marley, in press

Guillot, T. 1999, Science, 286, 72

Guillot, T., Burrows, A., Hubbard, W. B., Lunine, J. I., \& Saumon, D. 1996, ApJL, 459, 35

Hansen, J. E., \& Travis, L. D. 1974, Space Sci. Rev., 16, 527

Henry, G. W., Baliunas, S. L., Donahue, R. A., Fekel, F. C., \& Soon, W. H. 2000a, ApJ, in press

Henry, G. W., Marcy, G. W., Butler, R. P., \& Vogt, S. S. 2000b, ApJ, 529, L41

Henry, G. W., Baliunas, S. L., Donahue, R. A., Soon, W. H, \& Saar, S. H. 1997, ApJ, 474, 503

Hilton, J. L. in Explanatory Supplement to the Astronomical Almanac, ed. P. K. Seidelmann, (Mill Valley: University Science Books), p. 383

Hovenier, J. W., \& Hage, J. I. 1989, A\&A, 214, 391

Huovelin, J., Linnaluoto, S., Tuominen, I., \& Virtanen, H. 1989, A\&AS, 78, 129

Husson, N., Bonnet, B., Scott, N. A., \& Chedin, A. 1994, JQSRT, 48, 509

Karkoschka, E. 1994, Icarus, 111, 174

Koike, C., Kaito, C., Yamamoto, T., Shibai, H., Kimura, S., \& Suto, H. 1995, Icarus, 114, 203

Kurucz, R. 1992, in IAU Symp. 159, Stellar Population of Galaxies, ed. B. Barbuy \& A. Renzini (Dorderecht: Kluwer), 225

Ludwig, C. B. 1971, Appl. Opt., 10, 1057

Lunine, J. L., Hubbard, W. B., Burrows, A., Wang, Y-P, \& Garlow, K. 1989, ApJ, 338, 314

Marley, M. S., Gelino, C., Stephens, D., Lunine, J., \& Freedman, R. 1999, ApJ, 513, 879

Mathisen, R., Photo Cross-sections for Stellar Atmosphere Calculations - Compilation of References and Data, Inst. of Theoret. Astrophys. Univ. of Oslo, Publ. Series No. 1.

Matthews, J. M. 1997, http://www.astro.ubc.ca/E-Cass/VE-98/matthews/index.htm

Mayor, M., Naef, D., Udry, S., Santos, N., Queloz, D., Melo, C., \& Confino, B. 1999, http://obswww.unige.ch/ udry/planet/hd75289_ann.htm

Mayor, M., \& Queloz, D. 1995, Nature, 378, 355

Mazeh, T., Naef, D., Torres, G., Latham, D. W., Mayor, M., Beuzit, J-L., Brown, T., Buchhave, L., Burnet, M., Carney, B. W., Charbonneau, D., Drukier, G. A., Laird, J. B., Pepe, F., Perrier, C., Queloz, D., Santos, N. C., Sivan, J-P., Udry, S., \& Zucker, S. 2000, ApJ, 532, L55

Mishchenko, M., Travis, L. D., Kahn, R. A., \& West, R. A. 1997, JGR, 192, 16831

Petaev, M. I., \& Wood, J. A. 1998, Met. \& Plan. Sci., 33, 1123

Radzig, A. A., \& Smirnov, B. M. 1985, Reference Data on Atoms, Molecules, and Ions (Berlin: Springer-Verlag)

Russell, H.N. 1916, ApJ, 43, 173.

Saumon, D., Hubbard, W. B., Burrows, A., Guillot, T., Lunine, J. L., \& Chabrier, G. 1996, ApJ, 460, 993

Schneider, J., http://cfa-www.harvard.edu/planets/encycl.htm 
Seager, S., 1999, PhD Thesis, Harvard University

Seager, S., \& Sasselov, D. D. 2000, ApJ, in press, astro-ph/9912241

Seager, S., \& Sasselov, D. D. 1998, ApJ, 502, L157

Sharp, C. M., \& Huebner, W. F. 1990, ApJS, 72, 417

Sudarsky, D., Burrows, A., \& Pinto, P. 1999, submitted to ApJ, astro-ph/9910504

Taylor, F., \& Irwin, P. G. J. 1999 Astr. \& Geophys., 40, 3-21

Tinney, C. G., Delfosse, X., Forveille, T., \& Allard, F. 1998, A\&A, 338, 1066

Tsuji, T., Ohnaka, K., \& Aoki, W. 1999, ApJ, 520, L119

Tsuji, T., Ohnaka, K., \& Aoki, W. 1996, A\&A, 305, L1

Van de Hulst, H. C. 1957, Light Scattering by Small Particles (New York: Dover)

White, W. B., Johnson, S. M., \& Danzig, G. B. 1958, J. Chem. Phys, 28, 751

Whitney, B. A. 1991, ApJS, 75, 1293

Whitney, B. A., \& Hartmann, L. 1993, ApJ, 402, 605

Whitney, B. A., \& Hartmann, L. 1992, ApJ, 395, 529

Whitney, B. A., Wolff, M., \& Clancy, R. T. 1999, "Monte carlo radiative transfer models for Mars," in The Fifth International Conference on Mars, Abstract \#6213, LPI Contribution No. 972, Lunar and Planetary Institute, Houston (CD-ROM)

This preprint was prepared with the AAS $\mathrm{LAT}_{\mathrm{E}} \mathrm{X}$ macros v4.0. 

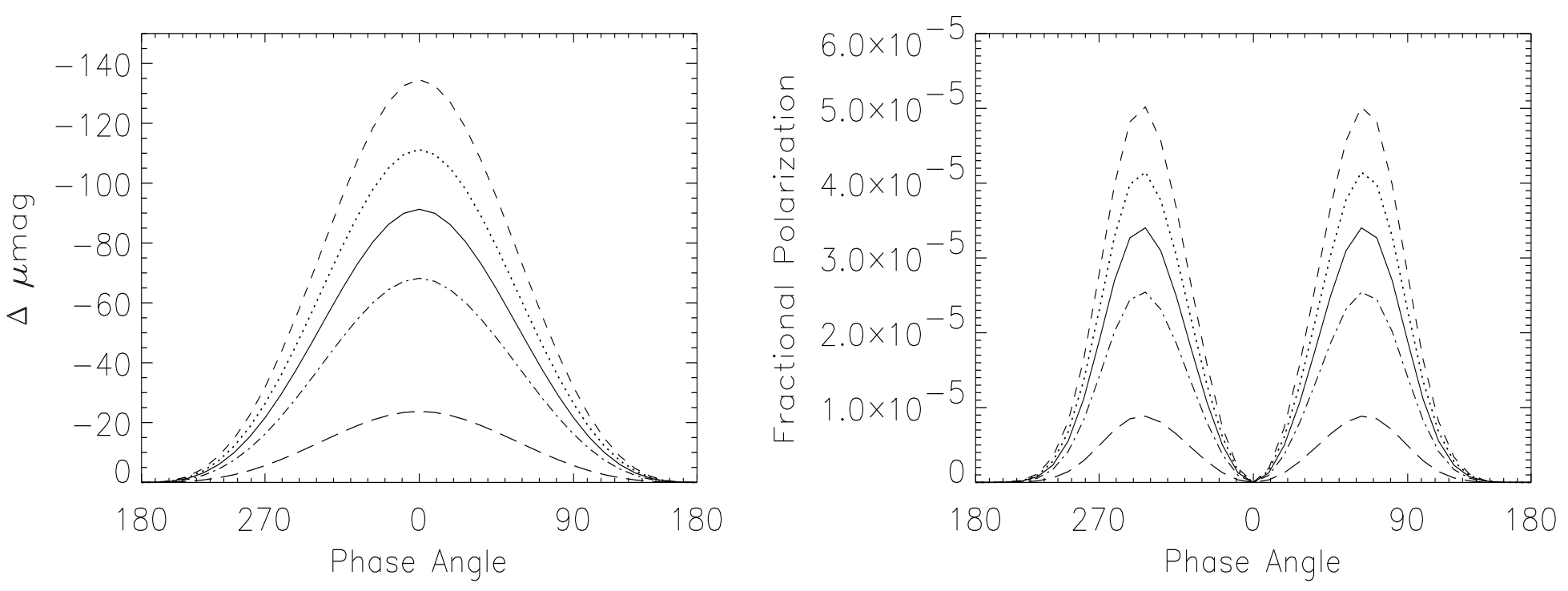

Fig. 1. - Lambert sphere light curves and polarization curves for CEGP systems with different $D$ and $R_{P}=1.2 R_{J}$. In descending order the curves are for $D=0.042 \mathrm{AU}$ (HD $187123 \mathrm{~b}$ ), $D=0.0462 \mathrm{AU}$ ( $\tau$ Boo b), $D=0.051 \mathrm{AU}(51 \mathrm{Peg} \mathrm{b}), D=0.059 \mathrm{AU}\left(v\right.$ And b), and $D=0.11 \mathrm{AU}(55 \mathrm{Cnc} \mathrm{b})$. For other $R_{P}$ the curves can be scaled - the light curves approximately and the polarization curves exactly - by the factor $\left(R_{P} / 1.2 R_{J}\right)^{2} . \Delta m=2.5 \mu \mathrm{mag}$ corresponds approximatley to a flux ratio of $10^{-6}$ (see equation (位).

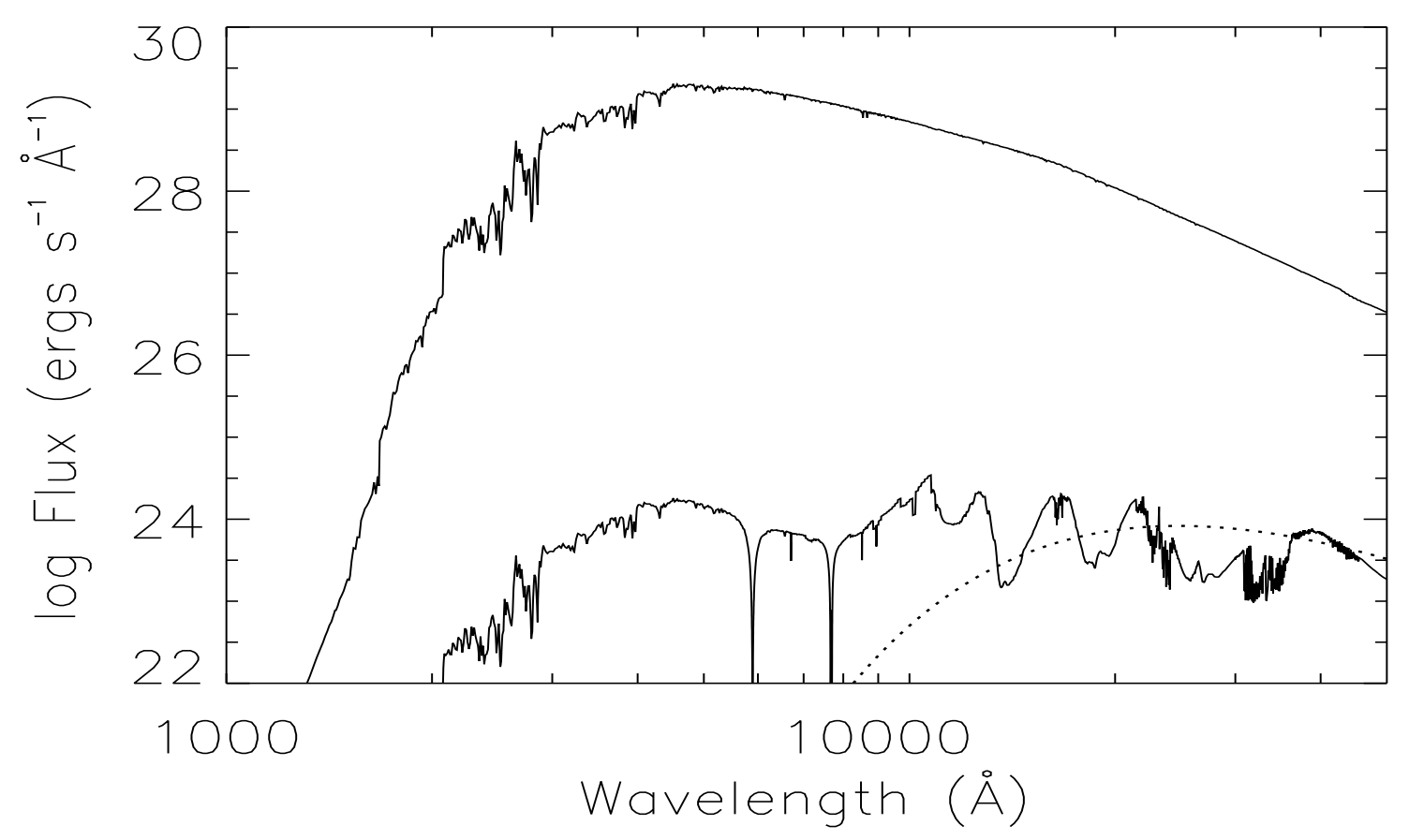

Fig. 2.- Flux of $51 \mathrm{Peg} \mathrm{A}$ and b. The upper curve is the model flux of $51 \mathrm{Peg} \mathrm{A}$ at the surface of the star, and the lower curve is the model flux of $51 \mathrm{Peg} \mathrm{b}$ with a homogenous $\mathrm{MgSiO}_{3}$ cloud with particles of $\bar{r}=0.01 \mu \mathrm{m}$. The dotted line is a blackbody with the same $T_{\text {eff }}$ as the planet model, $1170 \mathrm{~K}$. In 51 Peg b, the features in the blue and UV $(<5000 \AA)$ are reflected stellar features, the absorption features between $5000 \AA$ and $1 \mu \mathrm{m}$ are alkali metal lines from the planetary atmosphere, and the absorption features $>1 \mu \mathrm{m}$ are water and methane absorption bands. 


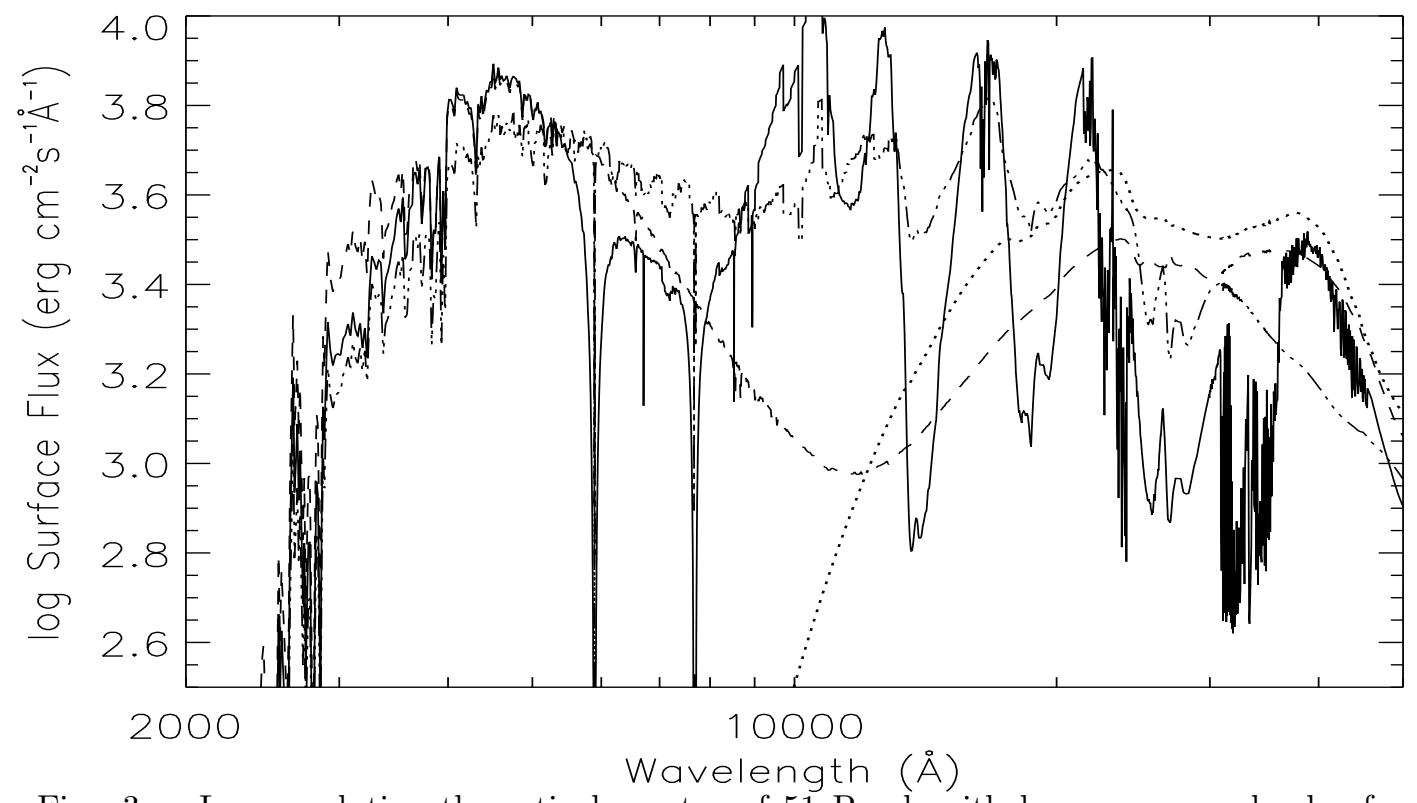

Fig. 3.- Low resolution theoretical spectra of $51 \mathrm{Peg} \mathrm{b}$ with homogeneous clouds of particles $\mathrm{MgSiO}_{3}-$ $\mathrm{Al}_{2} \mathrm{O}_{3}$-Fe in a gaussian size distribution. The dotted line is for particles with $\bar{r}=0.01 \mu \mathrm{m}$, dashed for $\bar{r}=0.1 \mu \mathrm{m}$, and dot-dash for $\bar{r}=10 \mu \mathrm{m}$. The solid line corresponds to the pure $\mathrm{MgSiO}_{3}$ cloud with particles with $\bar{r}=0.01 \mu \mathrm{m}$, shown on a different scale in Figure 2. The features in the blue and optical are reflected stellar features with the exception of the alkali metal lines. The $\mathrm{H}_{2} \mathrm{O}$ bands can be seen in the infrared. See text for details.

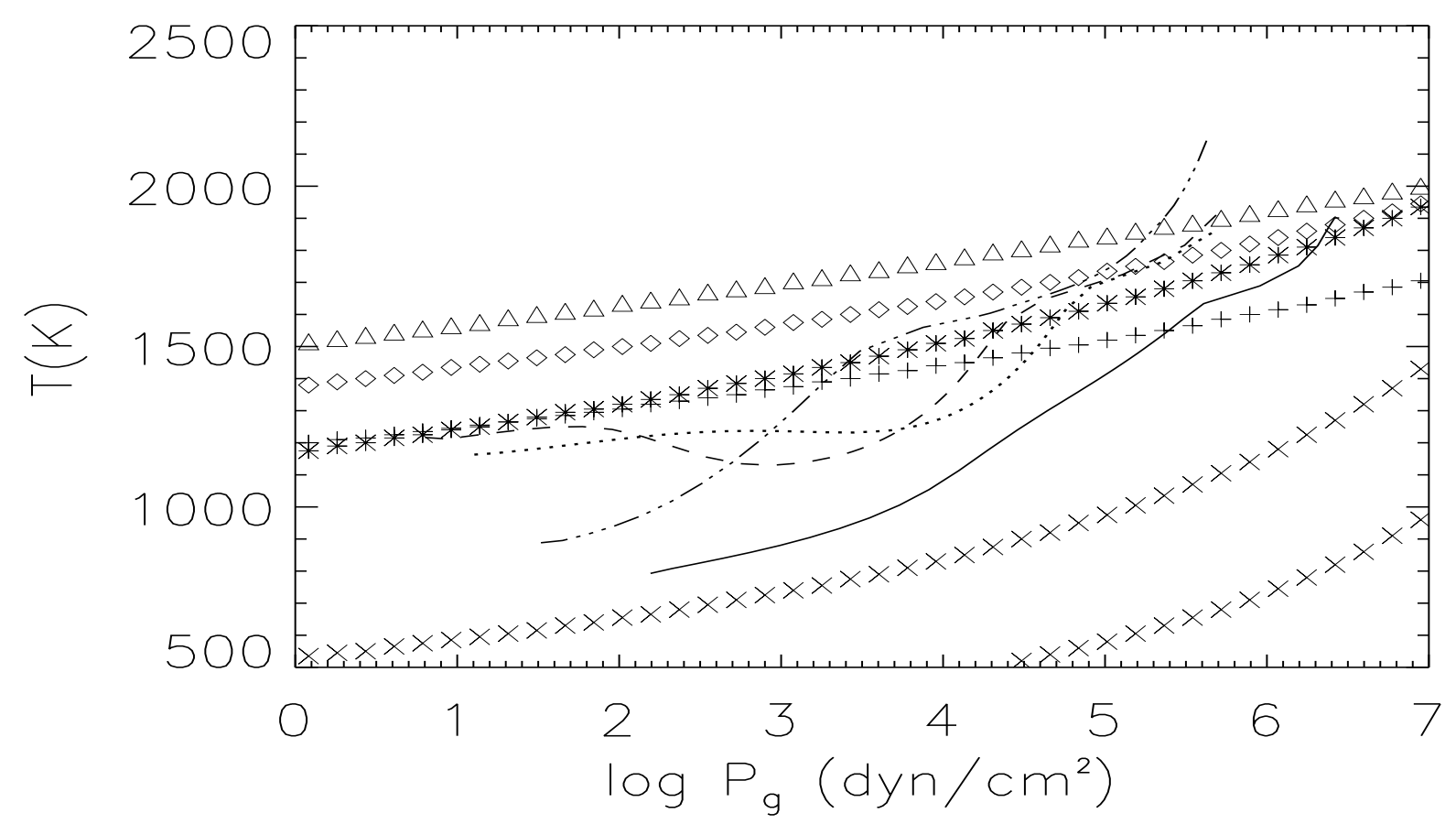

Fig. 4.- Temperature-pressure profiles for four different $51 \mathrm{Peg}$ b models. The models shown correspond to a homogeneous $\mathrm{MgSiO}_{3}-\mathrm{Al}_{2} \mathrm{O}_{3}$-Fe cloud with particles of $\bar{r}=0.01 \mu \mathrm{m}$ (dotted), $0.1 \mu \mathrm{m}$ (dashed), and $10 \mu \mathrm{m}$ (dot-dashed line). The solid line is a model with only $\mathrm{MgSiO}_{3}$ clouds with particles of $\bar{r}=0.01 \mu \mathrm{m}$. The symbols show the condensation curves of $\mathrm{CaTiO}_{3}$ (triangles), $\mathrm{Al}_{2} \mathrm{O}_{3}$ (diamonds), $\mathrm{Fe}(*), \mathrm{MgSiO}_{3}(+)$, the $\mathrm{CO} / \mathrm{CH}_{4}$ equilibrium curve (upper $\times$ ), and the $\mathrm{N}_{2} / \mathrm{NH}_{3}$ equilibirum curve (lower $\times$ ). The metallicity is $[\mathrm{Fe} / \mathrm{H}]=+0.21$, corresponding to that of $51 \mathrm{Peg} \mathrm{A}$. 


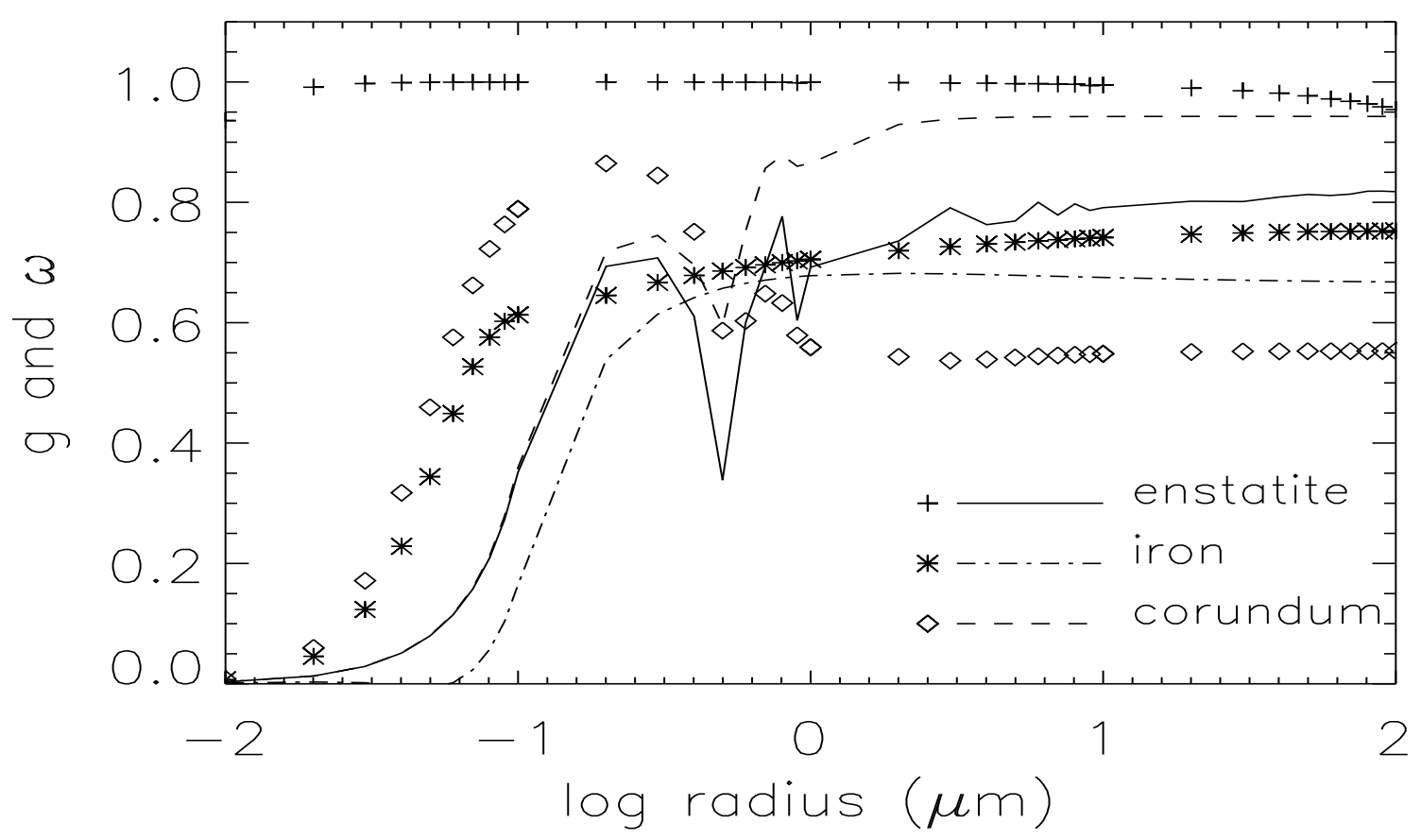

Fig. 5.- Scattering asymmetry parameter (lines) and single scattering albedo (symbols) for the three condensates used in this study. See discussion in text for details.
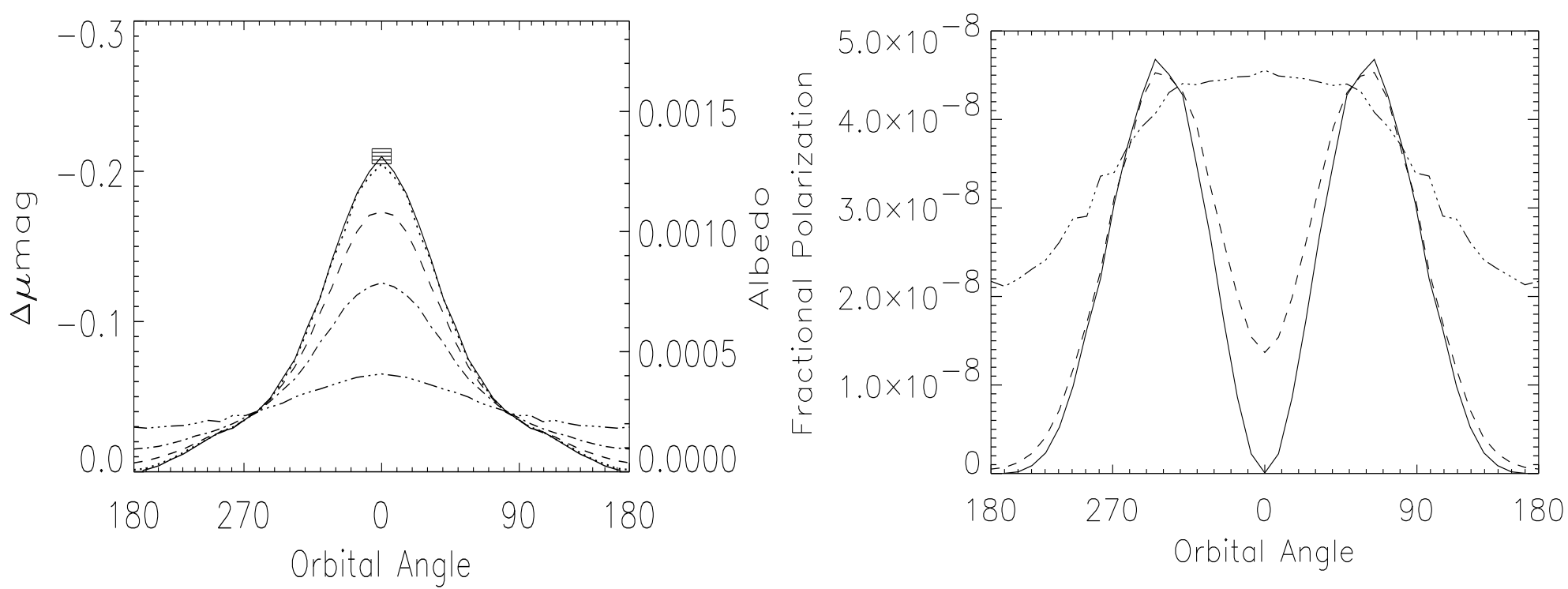

Fig. 6. - Light curves and fractional polarization for particles with $\bar{r}=0.01 \mu \mathrm{m}$. The lines correspond to different inclinations: solid $=90^{\circ}$, dotted $=82^{\circ}$, dashed $=66^{\circ}$, dash-dot $=48^{\circ}$, dash-dot-dot-dot $=21^{\circ}$. The hatched area is not observable; it represents the orbital angles at which the star is directly in front of the planet, which only occurs for $i>\theta_{T}$. For clarity the hatched area is not shown for the polarization fraction, and only three of the inclinations are shown. Fractional polarization at $i=21^{\circ}$ is noisier than at other inclinations because few photons scatter into these phase angles, as indicated by the light curve (left panel). 

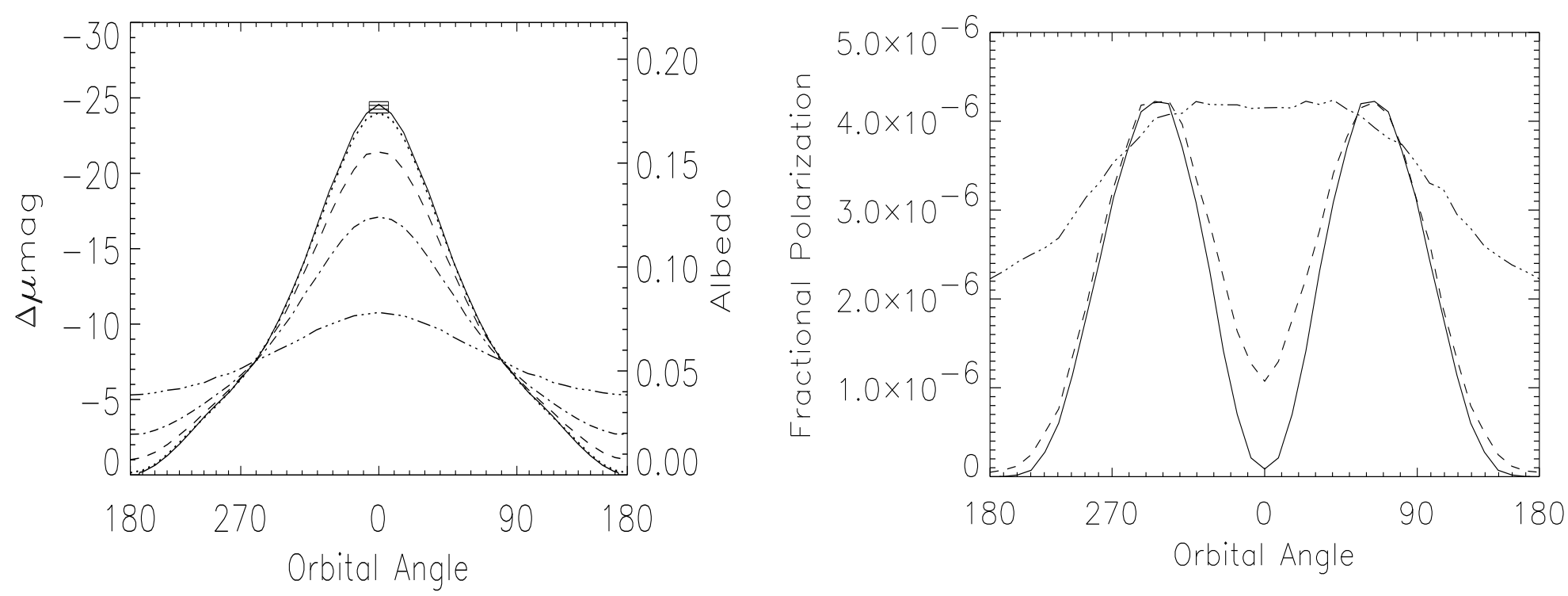

Fig. 7.- Light curves and fractional polarization for particles with $\bar{r}=0.1 \mu \mathrm{m}$. The curves are for the same inclinations as in Figure 6 .
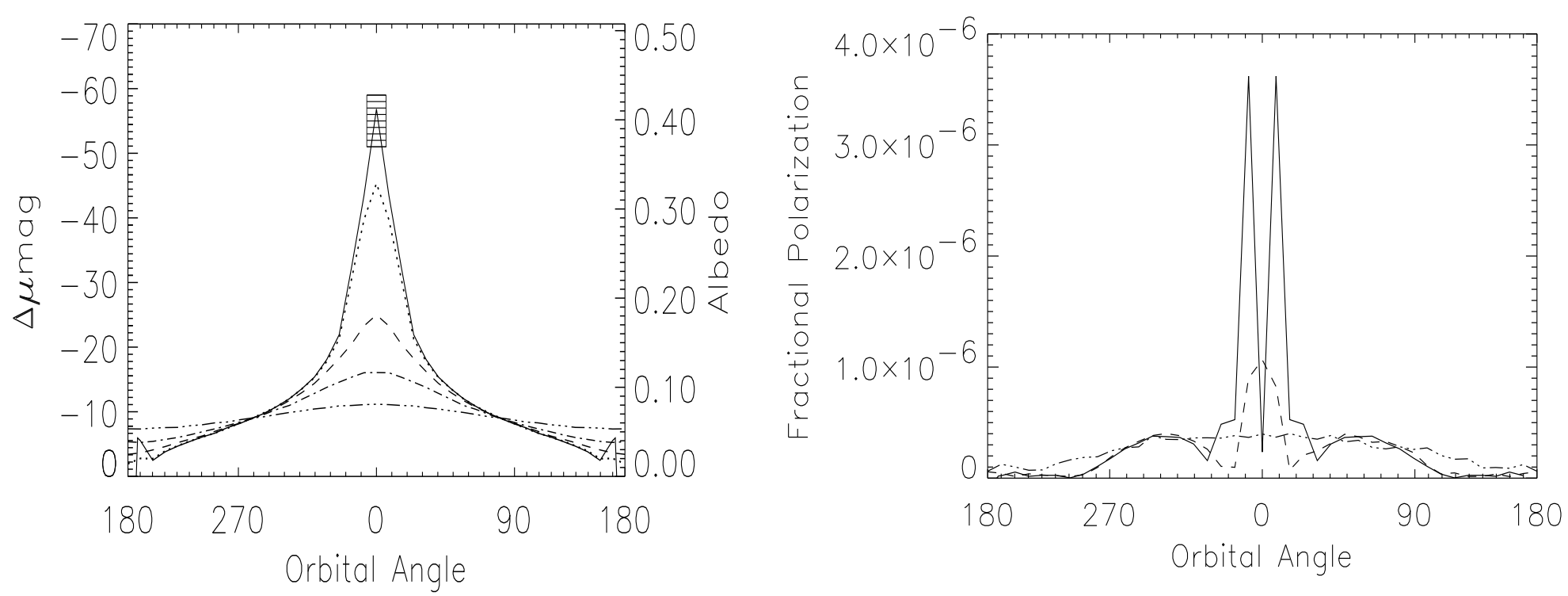

Fig. 8. - Light curves and fractional polarization for particles with $\bar{r}=1 \mu \mathrm{m}$. The curves are for the same inclinations as in Figure 6 . 

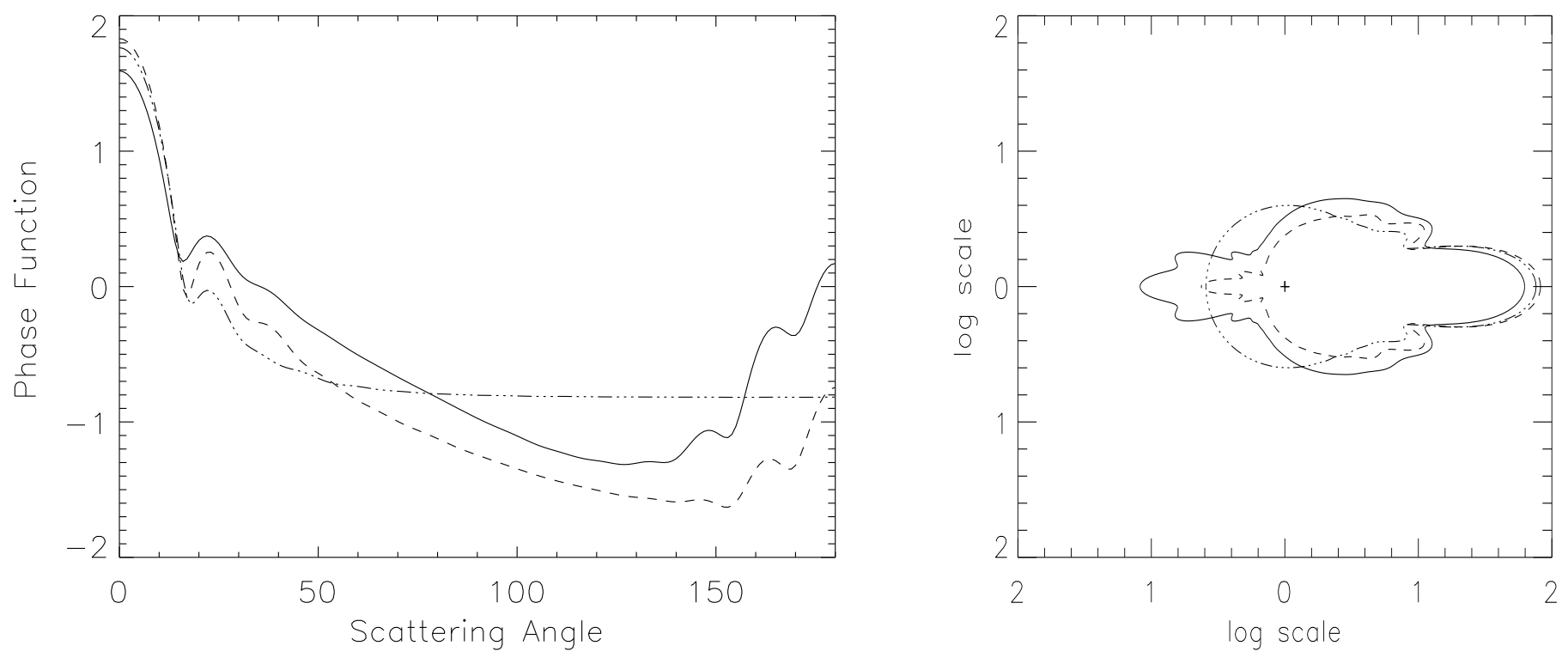

Fig. 9.- The phase functions and polar diagrams for the three condensates with $\bar{r}=1 \mu \mathrm{m}$. The solid line is $\mathrm{MgSiO}_{3}$, the dash-dot line $\mathrm{Fe}$, and the dashed line $\mathrm{Al}_{2} \mathrm{O}_{3}$. In the polar diagram the light is incoming from the left and the condensate particle is marked by the cross. The axes on the polar diagram are in a log scale, the units are dimensionless and only the relative numbers are important.
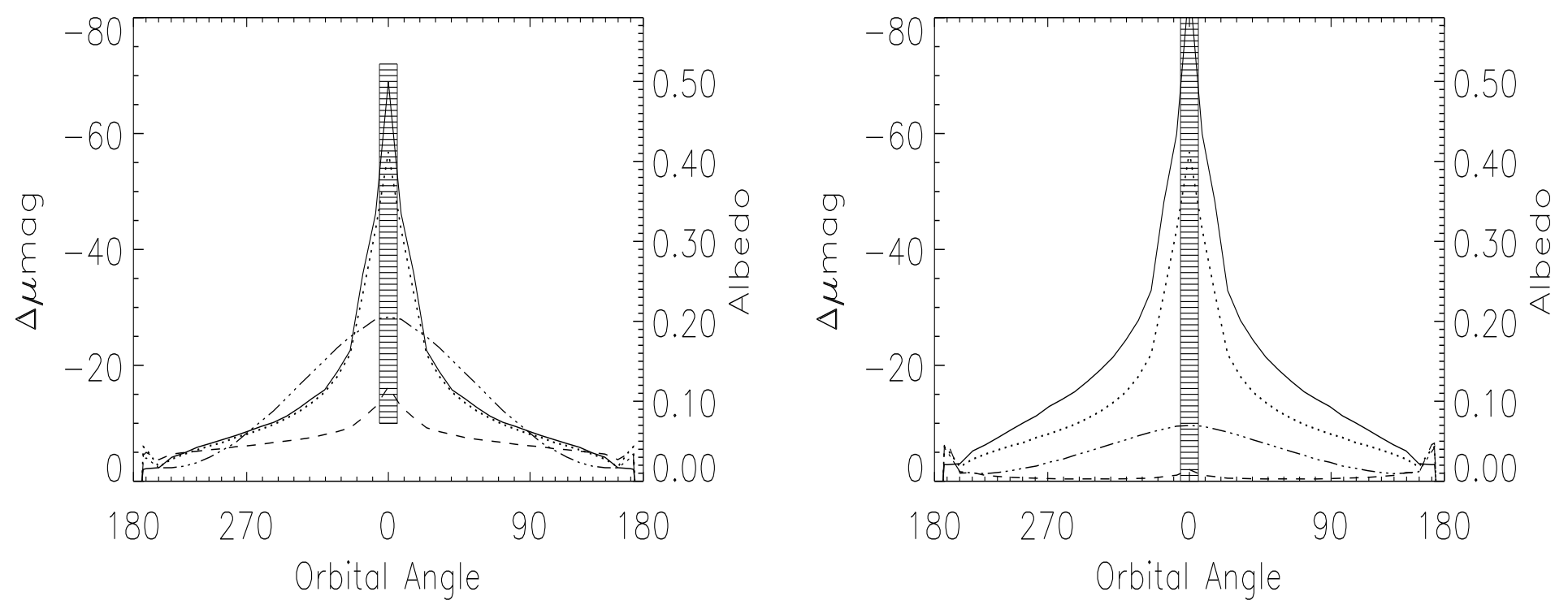

Fig. 10. - Light curves for $i=90^{\circ}$ for individual condensate particles with $\bar{r}=1 \mu \mathrm{m}$. The dotted lines are the light curves from the $\mathrm{MgSiO}_{3}-\mathrm{Fe}-\mathrm{Al}_{2} \mathrm{O}_{3}$ mix, the solid line is $\mathrm{MgSiO}_{3}$, dot-dash is $\mathrm{Fe}$, and dashed is $\mathrm{Al}_{2} \mathrm{O}_{3}$. Figure 10a shows the light curves as if each condensate was the only one present in the atmosphere. Figure 10 b shows the light curves for the phase function of each condensate, but with the same total opacity as in the $\mathrm{MgSiO}_{3}-\mathrm{Fe}-\mathrm{Al}_{2} \mathrm{O}_{3}$ mix. 

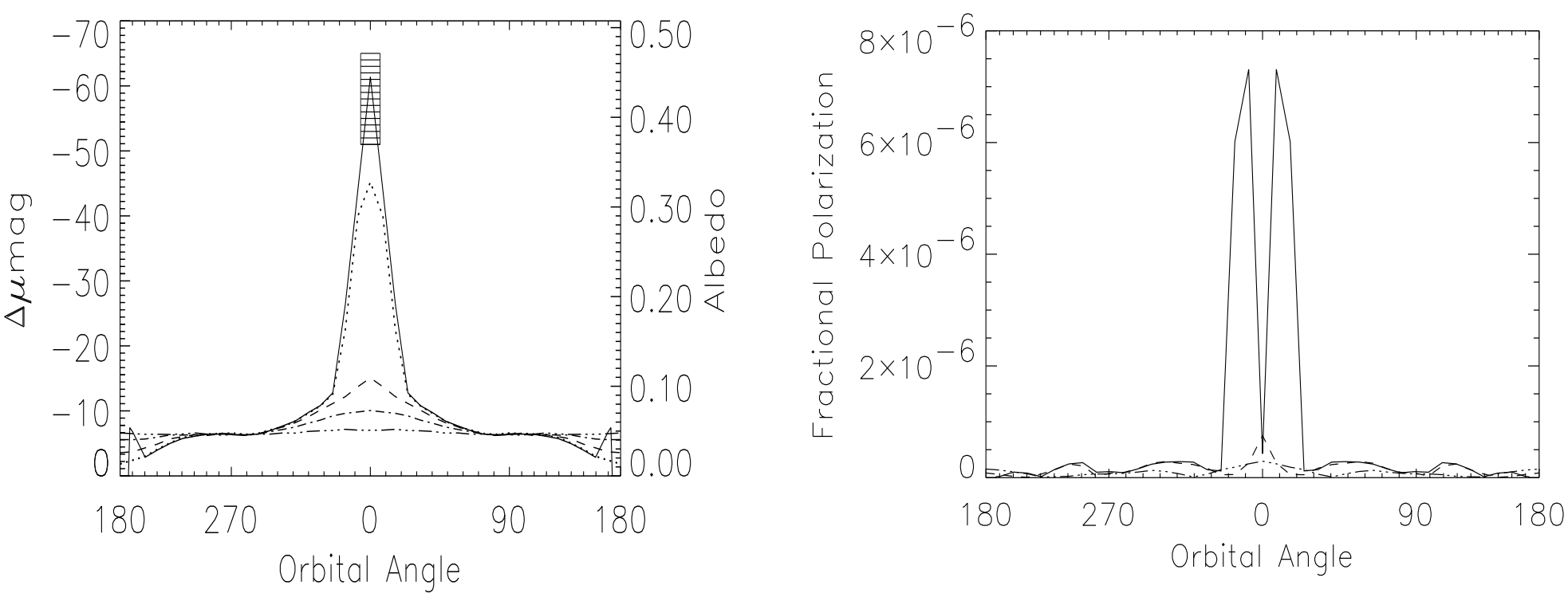

Fig. 11. - Light curves and fractional polarization for particles with $\bar{r}=10 \mu \mathrm{m}$. The curves are for the same inclinations as in Figure 6. The rise in the light curves at $\Theta>160^{\circ}$ is from light forward scattering through the upper atmosphere. Part of the transit light curve is visible near $\Theta=180^{\circ}$.
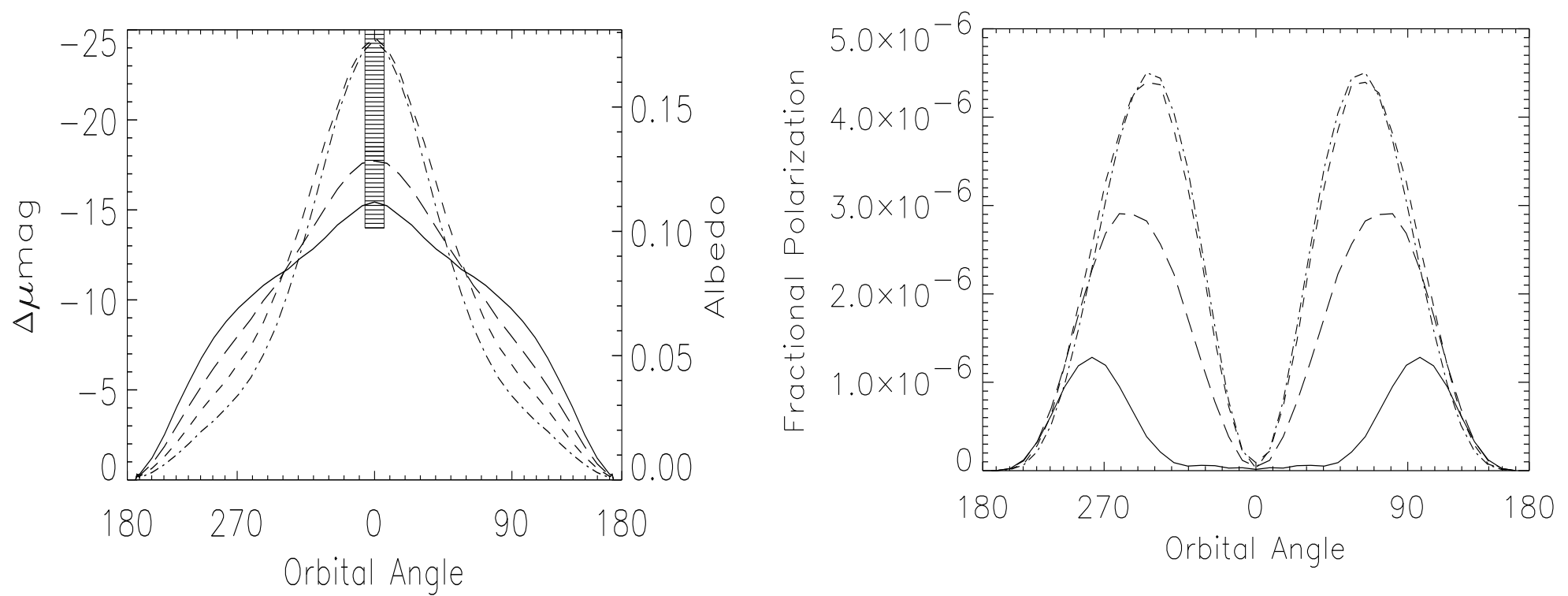

Fig. 12. - Light curves and fractional polarization at $i=90^{\circ}$ for condensates with $\bar{r}=0.1 \mu \mathrm{m}$, for $\mathrm{U}$ (solid), B (long dash), V (dash), and R(dot-dash). 


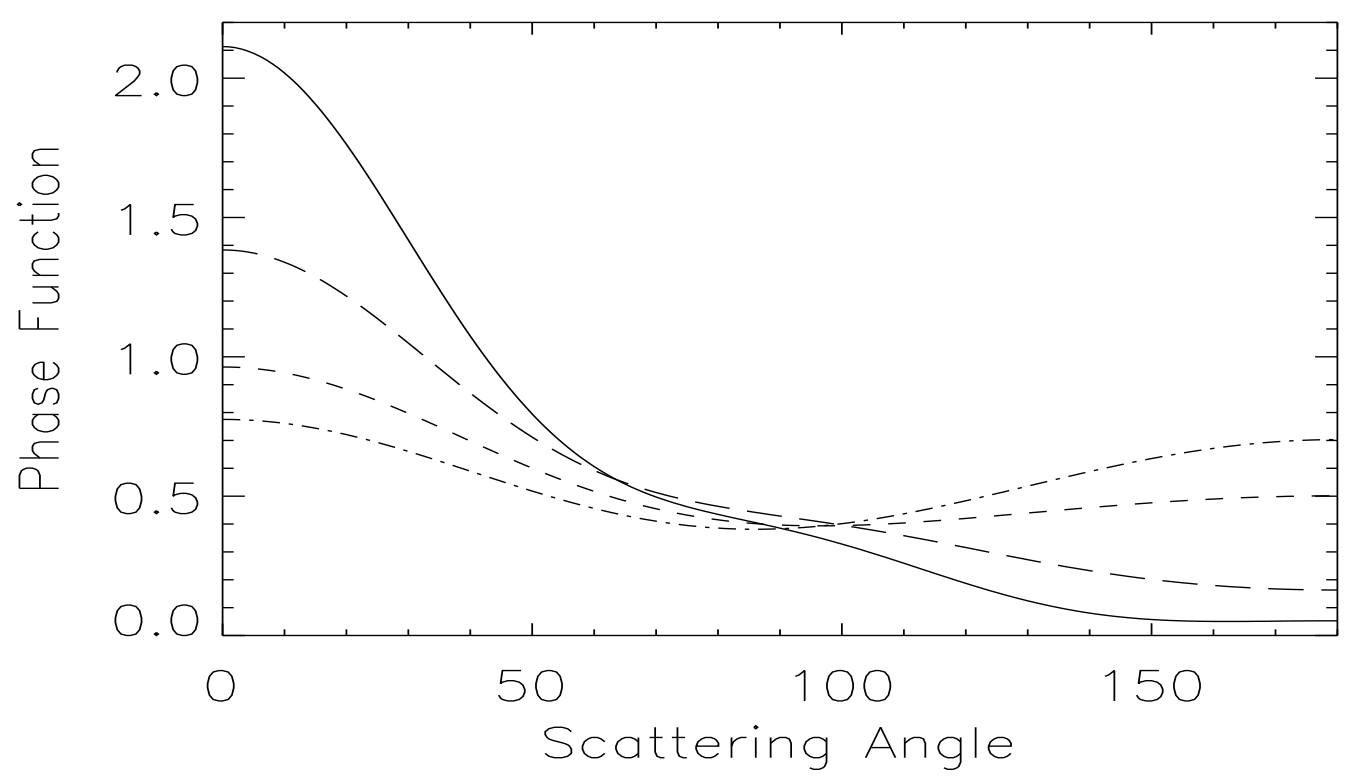

Fig. 13.- Phase function for Fe, for particles with $\bar{r}=0.1 \mu \mathrm{m}$, for $\mathrm{U}$ (solid), B (long dash), V (dash), $\mathrm{R}($ dot-dash).

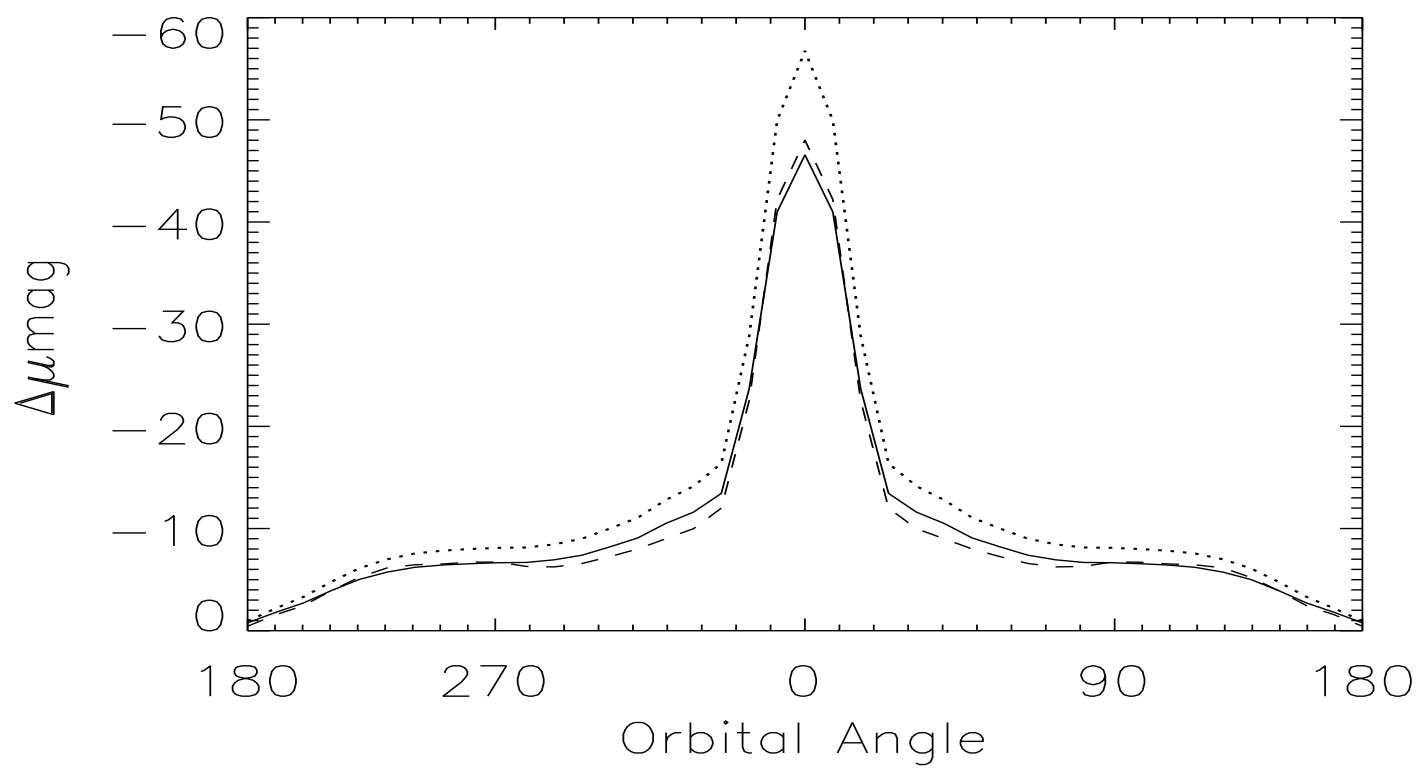

Fig. 14.- Predicted light curves for $51 \mathrm{Peg} \mathrm{b}$ (solid curve) and $\tau$ Boo $\mathrm{b}$ (dashed curve) for $i=82^{\circ}$ and particles with $\bar{r}=10 \mu \mathrm{m}$. The dotted curves is for a 51 Peg b-type planet with $D_{\tau B o o}$. 